\title{
“STELELE ȘI LALELELE”: SAGGIO DI MICROMONOGRAFIA STORICO-DESCRITTIVA DI UNA CLASSE FLESSIVA DELLA LINGUA ROMENA (II)
}

\author{
ROBERTO MERLO 1
}

\begin{abstract}
Stelele și Lalelele": Micromonography of an Inflectional Class in the Romanian Language (II). This article is part of a series aimed at reconstructing the history, and discussing the current state of what has been considered, from a Romance perspective, a peculiarity of Romanian language: the existence of an inflectional class of feminine nouns ending in tonic vowel (in short: $F \sqrt{V}-\emptyset$ ), which form the plural with the addition of the -le morpheme. The present paper, the second in the series, discusses some morphological traits of $F \sqrt{V}-\varnothing$ on the basis of a lexicographical corpus of contemporary standard Romanian: division in subclasses, internal morphological structure of its members (primitive and derivatives nouns, in particular diminutives, internal formations), and morphological variability.
\end{abstract}

Keywords: nominal morphology, Romance plural, Romanian language, Turkish loanwords, inflectional morphology

REZUMAT. “Stelele și lalelele”: încercare de micromonografie istoric-descriptivă asupra unei clase flexionare a limbii române (II). Acest articol face parte dintr-o serie care vizează discutarea istoriei și stării actuale a ceea ce s-a considerat, din perspectivă romanică, o particularitate a limbii române: existența unei clase flexionare de substantive feminine în vocală accentuată (pe scurt: $F \sqrt{ }$ v́- $\emptyset$ ), care formează genitiv-dativul singular și pluralul prin adăugarea morfemului -le. Prezentul articol, al doilea al seriei, discută câteva aspecte morfologice ale acestei clase pe baza unui corpus lexicografic de română contemporană standard: împărțirea ei în subclase, structura morfologică internă a substantivelor care fac parte din această clasă (substantive primitive și derivate, mai ales diminutive; formațiuni interne) și variabilitatea lor morfologică.

Cuvinte-cheie: morfologie nominală, plural romanic, limbă română, împrumuturi turcești, morfologie flexionară

1 Roberto MERLO è professore associato di Lingua e Letteratura romena presso il Dipartimento di Lingue e Letterature straniere e Culture moderne dell'Università di Torino. In ambito linguistico, la sua attività di ricerca è focalizzata sulla storia della lingua romena in epoca moderna e sui contatti romenoslavi. Email: roberto.merlo@unito.it. 
Il presente articolo ${ }^{2}$ costituisce la seconda parte di uno studio in più parti volto a ricostruire l'origine, lo sviluppo storico e lo stato attuale di quello che, in una prospettiva romanza, è spesso considerato un tratto distintivo della lingua romena: l'esistenza di una classe di s. F con morfema - $\emptyset$ al sg.NA e -le al sg.GD/pl (GLR $2,79-80)$, in breve: “F $\sqrt{\mathrm{V}-} \varnothing$ ”.

Nella prima parte di questo studio (Merlo 2020) ho illustrato la specificità di r. $\mathrm{F} \sqrt{\mathrm{V}}-\varnothing$ in ambito romanzo (evidenziando in particolare come il morfema $\mathrm{r}$. F sg.GD/pl -le non trovi corrispondenti immediati nelle altre lingue romanze, nella fattispecie nei dialetti italo-romanzi e in particolare in it., in cui i restanti morfemi r. di pl trovano un corrispondente storico e funzionale immediato) e ho ricostruito l'origine e l'evoluzione di questa classe dal lat. dan. fino ai dialetti $r$. storicamente attestati. Il mutamento fonetico chiave è lat. LL [ī] > [w] / V́_[a] (Sala 1976: 87-90, 136-170; ILR 2018, 366-367), per cui si viene a formare in

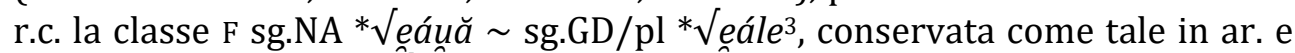
mr. ed "abbreviata" in $\sqrt{e}$ á in dr. (dove però a livello reg. si conserva anche la forma etimologica $\sqrt{e}$ áuă $)$ e ir. (dove $\sqrt{e}$ é evolve ulteriormente a $\sqrt{e}$ ):

(38)

\begin{tabular}{|c|c|c|c|c|c|}
\hline & LAT. & LAT. DAN. & R.C. & \multicolumn{2}{|c|}{ R. } \\
\hline \multirow{3}{*}{ sg } & $\sqrt{\breve{E} L L A}$ & $* \sqrt{\varepsilon} \bar{l} \bar{l}$ & \multirow{3}{*}{ *Ve e्áŭă } & \multirow{2}{*}{$>\sqrt{e}$ éa $;>$ ir. $\sqrt{e}$} & \multirow{2}{*}{ dr., ir. } \\
\hline & $\sqrt{\mathrm{E} L L A}$ & \multirow{2}{*}{$* \sqrt{\text { éla }}$} & & & \\
\hline & 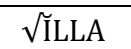 & & & $=\sqrt{e}$ éúă & ar., mr. \\
\hline \multirow{3}{*}{ pl } & $\sqrt{\breve{E} L L A E}$ & $* \sqrt{\varepsilon} \bar{l} \bar{l}$ & \multirow{3}{*}{ *V e éále } & \multirow{2}{*}{ > Véle } & \multirow{2}{*}{ dr., ir. } \\
\hline & $\sqrt{\mathrm{E} L L A E}$ & \multirow{2}{*}{$* \sqrt{ }$ éle } & & & \\
\hline & $\sqrt{\text { ILLLAE }}$ & & & $=\sqrt{e}$ éle & ar., $\mathrm{mr}$. \\
\hline
\end{tabular}

Sulla base della comparazione tra i dati offerti dai dial. r., infine, ho circoscritto (con tutte le approssimazioni del caso) un gruppo di vocaboli di eredità lat. in cui tale mutamento fonetico ha avuto luogo, andando a costituire

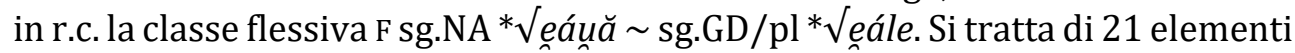
latini certi:

(39) 1. AGNĔLLA > dr. mia 'agnella', ar. ńauă, ir. ml'å; 2. *BABA > dr. bale (pl) 'bava', ar. bală, ar. bal'ă; 3. BUCCĔLLA > dr. bucea 'rivestimento metallico all'interno del mozzo di una ruota'; 4. CATĔLLA > dr. cățea 'cagna', ar., mr. cățauă; 5. CORRǏGIA o *CORĔLLA ( $\leftarrow$ cŏrium) (DELR, s.v.)

2 Ringrazio sentitamente i revisori anonimi per l'attenta lettura e le numerose e puntuali osservazioni, che hanno contribuito in maniera sostanziale a migliorare la versione finale di questo articolo.

3 La quasi totalità degli esempi sono $s$. in $\sqrt{\mathrm{E} L L A} \sqrt{\mathrm{E} L L A} \sqrt{\mathrm{I} L L A}$; cf. però anche $*$ PİLLA $\left(\leftarrow{ }^{*}\right.$ pillŭla) $>$ dr. piuă 'mortaio', in r.c. verosimilmente privo di pl o con pl = sg (Coteanu 1969, 41; per altri casi analoghi, cf. Merlo 2020, n. 34). Dai dati disponibili è emerso un unico etimo in $\sqrt{\text { ALLA, lat. }}$ RALLA menzionato da P. Papahagi per ar. ráŭ ${ }^{2}$ (DDA, s.v.) pl rále. 
> dr. curea 'cinghia; cintura', ar. cur(e)auă, mr. curauă; 6. DĪE > dr. zi 'giorno', ar. dzîuă, dzuă, mr. zuă, ir. zi (integrato in questa classe già in r.c.); 7. *DREPANĔLLA > dr. drepnea 'rondone'; 8. *FRONTICĔLLA > fruncea 'fronte', ar. frâmțeauă 'sopracciglio'; 9. *HIRUNDINĔLLA > dr. rândunea 'rondine'; 10. MARGĔLLA > dr. mărgea 'perl(in)a', ar. mărdzeauă, mr. măr(d)zeauă; 11. MAXĬLLA > dr. măsea 'molare', ar., mr. măseauă; 12. NOVĔLLA > dr. nuia 'giunco'; 13. PORCĔLLA > dr. purcea 'porcella', ar. purțeauă, mr. purtseauă, ir. purčę; 14. *PUSTĔLLA o *PUST(IC) ӖLLA > dr. pușchea 'vescica (nel cavo orale); afta'; 15. *RETĔLLA > dr. retea 'rete'; 16. SĔLLA > dr. șa, reg. șea 'sella', ar. ș(e)auă, mr. șauă; 17. STËLLA > dr. stea 'stella', ar., mr. steauă, ir. stę; 18. *SUBFRONTICĔLLA > dr. sufruncea 'ciglio', ar. $s(u)$ frâmțeauă 'sopracciglio'; 19. *SUMMICĔLLA o SENTICĔLLA > dr. smicea/sâmcea 'ramoscello'; 20. TURTURĔLLA > dr. turturea 'tortora', ar. turtureauă; 21. VITĔLLA > dr. vițea 'vitella', ar. vițauă, ir. vițé,

e 9 elementi probabilmente o possibilmente lat. ovvero mediati dal lat.:

(40) 22. sostrato (DELR, s.v.) > dr. argea; 23. *ENDRĔLLA (< lat. < gr. éndrion o sostrato, DELR; improbabile l'etimo tc. öyendere, DAD, s.v. andreáŭ $\breve{a})>$ dr. andrea (în-, un-) 'ferro da calza', ar., mr. andreauă; 24. *FUNICĔLLA > funicea 'funicella' (o dim. di funie 'fune', ILR 2018, 554); 25. *FURCĔLLA o FURCĬLLA > dr. furcea 'piccola forca' (o dim. di furcă 'forca (nella filatura)'); 26. OLLICĚLLA > dr. ulcea 'orciolo' (o dim. di oală 'olla', ILR 2018, 562); 27. VALLICĔLLA > dr. vâlcea 'piccola valle' (o dim. di vale'valle'); 28. *VESSICĔLLA > dr. reg. beștea 'vescica sulla lingua' (o da beșicea 'vescicuzza', ILR 2018, 551); 29. *VIRGĔLLA > dr. vergea 'vergella', ar. virdzeauă, mr. vir(d)zeauă (o dim. di vargă 'verga', ILR 2018, 562); 30. ZABA (< lat. < gr. zaba, CDER, o sostr., Borangic, Paliga 2013, in part. 7-9) > dr. $z a$ 'anello di una catena' (o dal medio greco, ILR 2018, 549).

In questa seconda parte mi focalizzerò sul dr., analizzando il profilo morfologico ed etimologico di $\mathrm{F} \sqrt{\mathrm{V}}-\emptyset$ in $\mathrm{dr}$. contemporaneo standard a partire da un corpus lessicografico appositamente stabilito.

Errata corrige. In chiusura del precedente articolo (Merlo 2020, 276) affermavo a proposito dei s. dr. $\mathrm{F} \sqrt{\mathrm{V}}-\varnothing$ di origine latina: "Anche includendo $\mathrm{i}$ termini di origine lat. meno sicura, i 30 termini elencati [...] non rappresentano che lo $0,4 \%$ delle 6.808 parole lat. ereditate in r. contemplate in ILR (2018, 494-537)". In realtà, il numero totale delle parole r. certamente, probabilmente o possibilmente ereditate dal latino elencate nel luogo citato non è "6.808" (cifra errata, dovuta a una mia svista redazionale) bensì 2.402, come segue: 1.751 parole 
r. di eredità latina certa, 1.588 con etimo unico ("cuvinte cert moștenite cu etimon necontroversat"; ibidem, 494-537) e 163 con più etimi possibili ("cuvinte cert moștenite cu etimon controversat"; ibid., 537-543), e 651 parole di eredità latina discussa, 192 con proposte etimologiche che vanno dall'eredità latina alla formazione interna al prestito ("cuvinte a căror origine latină nu este unanim recunoscută"; ibid., 543-549) e 459 (la maggior parte presenti solo in dr.) considerate vuoi di eredità diretta vuoi formazioni interne ("cuvinte moștenite sau formații românești”; ibid., 549-563). Di conseguenza, la rispettiva percentuale non è " $0,4 \%$ " bensì $1,2 \%$, una quota comunque molto ridotta.

4. $F \sqrt{ } \mathbf{V}-\emptyset$ in $\mathbf{r}$. attuale - profilo morfologico. Rispetto alla situazione originaria ricostruita per il r. c., in (d)r. attuale $F \sqrt{ } \mathrm{V}-\varnothing$ è arrivato a comprendere un numero assai più elevato di termini: lo spoglio di DEX16 e D00M2 conduce infatti per il romeno contemporaneo standard alla costituzione di un corpus lessicale di ben 383 termini appartenenti a $F \sqrt{V}-\emptyset^{4}$. Sulla base di questo corpus, in quanto segue analizzerò il profilo morfologico di $F \sqrt{ }$ - $-\varnothing$ in (d)r. attuale, limitando a quanto necessario per lo scopo del presente articolo la discussione etimologica, che sarà oggetto di analisi distesa nello studio successivo della presente serie.

4.1. Sottoclassi. Dal punto di vista del comportamento morfologico (formazione di sg.GD/pl), a seconda di quale sia il segmento vocalico finale di radice $\mathrm{F} \sqrt{\mathrm{v}-} \varnothing$ comprende 2 sottoclassi principali: vocale /'a/ ( $\mathrm{F} \sqrt{a}-\varnothing)$, dittongo /'ea/ $(\mathrm{F} \sqrt{ }$ éá-Ø), più l'unico $\mathrm{s}$. $\mathrm{F}$ in /'i/, zi $(\mathrm{F} \sqrt{i}-\emptyset)$. Le grammatiche descrittive contemplano di norma una terza sottoclasse, comprendente i s. con radice terminata in dittongo /'ia/ $\left(\mathrm{F} \sqrt{i}{ }_{n} a ́-\varnothing\right)^{5}$, la cui autonomia è tuttavia discutibile (cfr. infra, §4.1.3).

4.1.1. $\boldsymbol{F} \sqrt{a}-\boldsymbol{\emptyset}$. Questo sottotipo comprende s. F con radice in /'a/, che formano il sg.GD/pl semplicemente aggiungendo il morfema -le: es. sg.NA basmá- $\emptyset \sim$ sg.GD/pl basmá-le. Appartengono a questo sottotipo 137 s. su 383, oltre un terzo dei sostantivi presenti nel corpus (per la precisione il 35,77\%), di cui 15 presenti nel corpus solo (13 s.) o soprattutto ( $2 \mathrm{~s}$.) al pl e 6 solo al sg6:

4 Il corpus completo e i criteri della sua compilazione saranno pubblicati in chiusura della serie.

5 Uso /i/ invece di /j/ per evitare la confusione con il grafema $\langle\mathrm{j}\rangle$ in trascrizioni parziali quali

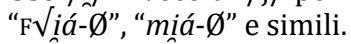

6 Nelle liste seguenti indico in corsivo grassetto le var. principali e in corsivo le var. secondarie di rilevanza morfologica (per la variazione morfologica inter- e intraclasse di $\mathrm{F} \sqrt{\mathrm{V}-} \emptyset \mathrm{cf}$. infra, $\S 4.3)$. Non indico qui le varianti puramente fonetiche; nel caso di omonimi preciso il senso alla prima occorrenza tra parentesi tonde. 
(41) aba, aga, alpaca ${ }^{1}$ (lega), alpaca (animale) $^{7}$, ana, anafora, angara, automacara, baba, bacara $^{1}$ (cristallo), bacara $^{2}$ (gioco di carte), baclava, baga (sg), balama, bale (pl), basma, baș-aga, beșli-aga, bina, cacealma, caimacam-aga, cana ${ }^{1}$ (cannello), canale ${ }^{2}$ (pl) (fiori), canara, canava, capama (sg), caplama, capudan-/căpitan-pașa, cara, cardama, carvasara, cașa (sg), cazma, cealma, cherhana, ciulama, cosmeticale (pl), cumbara, curama, dambla, dandana, danga, dara, debara, diamanticale (pl), falbala, farfara, furda, geamparale (pl), giuvaiericale (pl), haimana, halca, halima, halva, haraba, harșa, hazna, iaca ${ }^{1}$ (colletto), iaca ${ }^{2}$ (sg) (tabacco), iavașa, iofca, istericale (pl), lamba, locma, maca, macara, mahala, mala, mangafa, manta, marda, masala, mascara, mola, mucava, musaca, mușama, nacafa, nevrica (+pl), nicochera, novita, nuga, oca, orta, osanale (pl), pafta, panama ${ }^{1}$ (intreccio; tessuto), panama 2 (imbroglio), para, pașa, patalama, pijama, politica (pl), pristanda, protipenda, pușlama, saca, sama, sanda, sarma, sarsana, satara, sofa, sofra, sosma, șabana, șale (pl), șandrama, șasla, tabla, tabulhana, tacla $(+\mathrm{pl})$, tafta, taiga, tanana (sg), tangara, tara, tarama (sg), tarapana, tarara, tarla, tasma, tava, trandada, trufanda, tura, tuslama, ulema, urale (pl), za, zagara, zaharicale (pl), zalhana, zarpa, zenana, zumaricale (pl), zurba.

Come si vedrà più in dettaglio nell'analisi del profilo etimologico, questo sottotipo è costituito per la quasi totalità da prestiti (dal gr., dal fr. e soprattutto - per oltre la metà - dal tc.) ${ }^{8}$.

4.1.2. $F \sqrt{\text { eá- }}$ Ø. Questo sottotipo comprende i s. F terminati in dittongo /'êa/, il cui sg.GD/pl si forma con l'aggiunta di -le e l'applicazione dell'alternanza fonetica /'ea/ > /'e/: es. sg.NA steá-Ø sg.GD/pl sté-le (cf. Merlo 2020, 268) ${ }^{9}$. Appartengono $\mathrm{F} \sqrt{e}$ é- $\emptyset$ oltre la metà dei sostantivi presenti nel corpus, $226 \mathrm{~s}$. (pari al 59\%), dei quali 11 registrati nel corpus solo (10 s.) o soprattutto (1 s.) al pl e 5 solo al sg:

7 La numerazione dei lemmi è quella adottata nelle fonti lessicografche che si trovano alla base del corpus.

8 A livello storico gli unici s. di eredità lat. appartenenti $a b$ origine a questo sottotipo sono sg *bauă / $b a<$ *BABA e prob. za, che hanno sviluppato i pl analogici bale e zale; allo stesso modo, qualora si consideri șale (pl) 'lombi' pl di șa 'sella', come in realtà è dal punto di vista etimologico, si potrebbe includere in $\mathrm{F} \sqrt{a}-\emptyset$ anche il paradigma sg șa $\sim$ pl șale (< SËLLA, SĔLLAE). Sulla base di simili considerazioni di natura diacronica ho incluso nel corpus di s. $\mathrm{F} \sqrt{\hat{\mathrm{V}}}-\emptyset$ bale $(\mathrm{pl})$ e șale $(\mathrm{pl})$, ancorché DEX16 e DOOM2 li registrino come pluralia tantum (quest'ultimo separato e indipendente da F sg șa 'sella' pl șei), così come altri s. registrati come pluralia tantum e per cui spesso neppure altre opere lessicografice registrano forme di sg, es. giuvaiericale $(\mathrm{pl})$, adormițele $(\mathrm{pl})$ ecc. Ho ovviamente incluso i singularia tantum in quanto il morfema -le marca anche il sg.GD.

9 Secondo DEX16 e DOOM2, vopsea è l'unico s. FلV́- $\varnothing$ che oltre al pl regolare vopsé-le ha anche un pl (irregolare) vopsél-uri, di fatto riconducibile alla var. secondaria di sg vopseál-ă. 
(42) acadea, adormițele (pl), alagea, albăstrea, alunea, andrea, argea, baccea, bagea, bălticea, beizadea, belea, besactea, bezea, bidinea, boccea, brățea, brândușea, brebenea, bretea, brumărea, bucățea, bucea, cafea, cafenea, canapea, caramea, catifea, căpețea, cărticea, cățea, cecmegea, cercevea, cherestea, chiftea, chisea $^{1}$ (vaso), chisea ${ }^{2}$ (borsa), cișmea, citarea, ciumărea, ciuturea, codănea, corăbiele (pl), cordea, crengurea, crestătea, croazea (sg), crușătea, cucuvea, cupea, curea, dairea, derea, drepnea, duminecea, dungățea, dușumea, fălcea, fărâmele (pl), fântânea, feregea, fermenea, fesfesele (pl), fidea (sg), flanea, floricea, friganea, gălbenea, găurea, gebea, gevrea, ghebea, ghermea, ghilimele (pl), ghiulea, ghizdea, giubea, giurumea, gogonea (+pl), gogoșea, grădea, grimea, halagea, havalea, hârlostea, imamea, ipingea, irmizea, îngerea, jaluzea, jartea, jucărea, lalea, lăcrimea, lerpenea (sg), libadea, lichea, lingurea ${ }^{1}$ (pianta), lingurea $^{2}(\mathrm{sg})$ (parte bassa dello sterno) ${ }^{10}$, livrea, lovele (pl), lulea, madea, mahmudea, malotea, manea, mărgărele (pl), mărgea, măsea, măturea, menghinea, merdenea, mertepea (sg), meterhanea, micșunea, mielărea, mielușea, militea, minarea, minciunea, miorea, muștea, narghilea, norea, ocheșele (pl), oprea, pacea, pangea, panglicea, pansea, parcea, patlagea, pavea, părticea, păsărea, pătrățea, peltea, percea, perdea, peruzea, picățea, pielicea, pingea, plămânărea, plăsea, podea, poezea, pogăcea, porumbea ${ }^{1}$ (frutto), porumbea ${ }^{2}$ (animale), potecea, preducea, proptea, protostea, pupezea, purcea, puschea, radiostea, rămurea, rândunea, refenea, regea, rețea, rindea, rocoțea, rogodele (pl), rondea, roșătea, rotoțele (pl), saftea, safterea, saltea, saragea, sardea, sălățea, sărățea, scândurea, schimbea, scordolea, secărea, smicea, soarea, sorbestrea, stea, străchinea, stricnea, surcea, șahzadea, șosea, șușanea, șușanea, tanea, tapangea, tâlhărea, tejghea, telegea, telelea, telemea, temenea, testea, teșcherea, tetrea, tinichea, turturea, tutea, ulcea, uscăturea, uscățea, vadea, vopsea $^{11}$, vâlcea, vântrea, vâzdogea, ventricea, vergea, verigea, vindecea, vinețea, viorea, vișinea, vițea, zaherea, zarnacadea, zăbrea, zeflemea, zorea.

In questa sottoclasse rientra la maggior parte dei s. originari di eredità lat. certa o probabile (18 s.: andrea, bucea, cățea, curea, drepnea, mărgea, măsea, purcea, pușchea, rândunea, rețea, smicea, stea, turturea, ulcea, vâlcea, vergea, vițea), i quali rappresentano però meno di un decimo dei s. totali della sottoclasse. Per il

${ }^{10}$ Facendo eccezione alla prassi di separare i lessemi in base all'etimologia (es. alpaca' 'alpaca' < fr. alpaca, alpaca ${ }^{2}$ 'alpacca' < td. Alpaka, chisea ' 'piccolo vaso di vetro, cristallo o porcellana in cui si tiene la confettura' < tc. kâse, chisea $^{2}$ 'borsa (in pelle) in cui si tengono soldi o tabacco' < tc. kese, kise) seguo D0OM2 nel trattare come lessemi distinti i due sensi di lingurea registrati nel corpus: 1 . 'cochlearia medicinale (Cochlearia officinalis)' e 2. 'parte bassa dello sterno', in quanto, sebbene in entrambi i casi si tratti di un der. di s. F lingură 'cucchiaio' (< lat. LINGŬLA), il primo si può collegare al senso primario della base (cf. anche lingurea 'cucchiaino' in MDA2, non presente nei dizionari su cui è basato il corpus) mentre il secondo, marcato come "pop.", è legato al senso specifico che questa assume nel sintagma reg. e pop. lingura pieptului (lett. 'il cucchiaio del petto') 'id.'.

11 Oltre alla var. principale vopsea, DEX16 include la var. fonetica văpsea e la var morfologica di quest'ultima văpseală, ma non la var. morfologica diretta vopseală (presente invece in MDA2): per comodità di esposizione, qui ho considerato var. principale vopsea e var. secondaria vopseală. 
resto, circa la metà è costituito da prestiti dal tc., e un terzo da formazioni interne: formati suffissati e composti, onomatopee e derivati regressivi (retroformazioni) (cf. infra, §4.3.1). In particolare, rientrano in $\mathrm{F} \sqrt{e}$ é- $\varnothing$, che numericamente è il sottotipo più consistente, un gran numero di suffissati a carattere diminutivale (cf. infra $\$ 4.2 .2 .1$ ).

4.1.3. F $\sqrt{i a ́}-\boldsymbol{\emptyset}$. Di norma le grammatiche romene contemplano l'esistenza di una terza sottoclasse di $\mathrm{F} \sqrt{\mathrm{V}}-\emptyset$, comprendenti i s. terminati in dittongo /'ia/ (cf. $\mathrm{GLR}_{2}, 80$ ), che formano il sg.GD/pl aggiungendo -le (con un'unica eccezione nel corpus, cf. infra) a volte con e a volte senza l'applicazione dell'alternanza fonetica /'ia/> /'ie/: es. sg.NA maiá- $\varnothing \sim$ sg.GD/pl maíné-le vs. sg.NA raiá- $\varnothing \sim$ sg.GD/pl raiá-le, senza che sia possibile predire in quali casi essa si applichi e in quali no. Apparterrebbero a questo sottotipo poche parole, 19 (il 4,96\% del totale), per i quali il corpus lessicografico analizzato registra $13 \mathrm{sg} . \mathrm{GD} / \mathrm{pl}$ con alternanza -12 regolarmente in $\sqrt{i}$ éele $(43 . a)$ più un unico s. singulare tantum con sg.GD irregolare (di tipo pop., cf. infra \$4.3.1) $\sqrt{i}$ é-li (43.b) - e 6 senza alternanza (43.c), di cui 1 registrato nel corpus con il solo sg:

(43) (a) boia, caia, ecpaia, maia, mia, muhaia, nisfia, nuia, paia, rubia, saia ${ }^{1}$

(filo), saia ${ }^{2}$ (ricovero), saia ${ }^{3}$ (tessuto);

(b) mumbaia;

(c) capuchehaia, chehaia, hataia (sg), raia, sermaia.

Da un punto di vista morfologico, tuttavia, tutti i s. usualmente inquadrati in questo sottotipo a sé potrebbero essere considerati radici in /'a/ o /'ea/ preceduti da / $\mathrm{i} / \mathrm{e}$ inseriti di conseguenza in uno dei due sottotipi precedenti in funzione della presenza o meno di fenomeni di alternanza fonetica. I s. che non presentano alternanza potrebbero infatti essere considerati s. di tipo $\mathrm{F} \sqrt{a}-\varnothing$ : es. sg.NA raiá- $\varnothing(=$ basmá- $\emptyset) \sim$ sg.GD/pl raiá-le (= basmá-le), mentre quelli in cui l'alternanza ha luogo potrebbero essere considerati s. di tipo $\mathrm{F} \sqrt{e} a ́-\emptyset$, in cui hanno luogo gli usuali passaggi */'iea/ > /'ia/ / _./a ə \#/ e */'iena/ > /'ie/ / _./e i

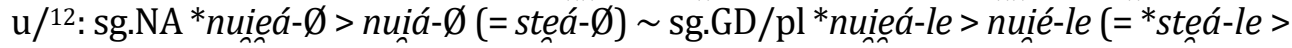
sté-le; cf. Merlo 2020, 268) ${ }^{13}$.

12 Ovvero */'iena/ > /'ín/ quando nella sillaba seguente è presente /ə/, /a/ o finale di parola (es. miá-Ø; storicamente, tuttavia, $\leftarrow$ miáuă < *mieáuă, cf. Merlo 2020, 270-274), e */'iea/ > /'ie/ quando nella sillaba seguente è presente $/ \mathrm{e} / \mathrm{j} / \mathrm{i} / \mathrm{o} / \mathrm{u} /$ (quest'ultima condizione, rilevante anche per /'ea/ > /'e/, vale solo per i s. F: es. F sg.NA seár-ă, steá- $\emptyset$, miá- $\emptyset$, treáb-ă, iárb-ă

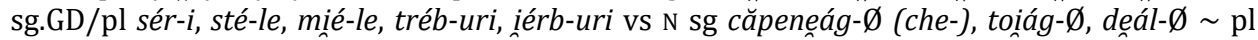
căpenég-e (che-), toiég-e, ma déál-uri; cf. anche Agard 1953, 138-139).

13 Eccezione parziale parrebbe sg.NA nisfia sg.GD/pl nisfiele, pronunciato secondo la norma ortoepica attuale /ni.sfi'a/ /ni.sfi'ele/ e quindi unico esempio di questo tipo in cui il dittongo 
In questo modo $\mathrm{F} \sqrt{\mathrm{V}}-\varnothing$ avrebbe solo due sottotipi, $\mathrm{F} \sqrt{a}-\varnothing$ con sg.GD/pl $\sqrt{a}-l e$ e $\mathrm{F} \sqrt{e} a^{\prime}-\varnothing$ con sg.GD/pl $\sqrt{e}-l e$ (eccezionalmente $\left.\sqrt{e}-l i\right)^{14}$. Attribuire i s. in /'ia/ all'una o all'altra in funzione del comportamento morfologico nella formazione del sg.GD/pl (assenza di alternanza vocalica: $\mathrm{F} \sqrt{a}-\emptyset$, presenza di alternanza vocalica: F $\sqrt{e a ́}-\emptyset)$, tuttavia, non risolve il problema dell'“impredicibilità", ma lo sposta semplicemente da questa all'identificazione della sottoclasse specifica di appartenenza. Poiché */'iea/- $\emptyset>/$ 'ia/- $\varnothing$, infatti, l'appartenenza di un s. F uscente in /'ia/ a $\mathrm{F} \sqrt{e}$ é- $\varnothing$ o a $\mathrm{F} \sqrt{a}-\varnothing$ non è decidibile sulla base della struttura fonomorfologica di superficie del sg.NA (né in base a fattori di altra natura, ad es. semantici o sociolinguistici: quasi tutti i s. di tipo /'ia/ sono cultismi e/o storicismi di origine tc.). Tale appartenenza è quindi codificata nel lessico.

4.2. Struttura morfologica. La questione della struttura morfologica è complessa, in quanto da un lato, in diacronia, essa è strettamente connessa alla ricerca etimologica (opzioni diverse relative all'origine di una parola possono implicare differenti analisi della sua struttura morfologica), e dall'altro, in sincronia, le analisi dei parlanti possono non corrispondere a quelle dei linguisti (tale che una parola viene segmentata in modo diverso da quello storicamente accertato; esemplare il caso degli pseudoalterati, cf. infra, §4.2.1.1). Nella classificazione che segue ho tenuto conto sia dell'etimologia sia di eventuali processi di risegmentazione e formazione interna, e ho escluso dall'analisi il piccolo gruppo di 14 s. con etimologia sconosciuta o incerta (baccea, ghermea, halagea, hârlostea, iaca ${ }^{2}$, lerpenea, militea, preducea, pristanda, saia ${ }^{1}$, sama, sorbestrea, tangara, trandada), per i quali è impossibile o rischioso pronunciarsi in merito all'eventuale struttura morfologica (es. baccea: $<$ tc. bahsızça = primitivo o < ${ }^{*}$ babcea $\leftarrow b a b a$ + -cea = derivato?; cf. DELR, s.v.; preducea: etimologia sconosciuta, cf. MDA2, o $\leftarrow$ v. preduci, cf. SDLR, CDER = derivato?).

4.2.1. Sostantivi primitivi. Dal punto di vista della struttura morfologica interna la soverchia maggioranza dei s. di tipo $\mathrm{F} \sqrt{\mathrm{V}}-\varnothing-286$ elementi, pari al $74,67 \%$ del totale - è costituita da s. primitivi, ovvero non creati a partire da parole già esistenti nella lingua:

/'ea/ non sarebbe preceduto da /i /; è tuttavia possibile postulare l'esistenza di una semivocale di raccordo [i] (v. l'interpretazione di m sg georgian [ḑe.or.dzi'jan] in Neamțu, Bocoș, Roman 2019, 311-313), tale per cui */ni.sfi'ęa/ = *[ni.sfi'iea $>$ [ni.sfi'ia $]=$ /ni.sfi'a/.

14 Tale analisi è per altro in accordo con la realtà dell'origine e dello sviluppo storico di questa classe, $\mathrm{F} \sqrt{e}$ é́- $\emptyset$ rappresentando il nucleo originario di $\mathrm{F} \sqrt{\mathrm{V}}-\varnothing$ (che include anche i due s. di eredità lat. in /'ia/: NOVĔLLA, AGNĔLLA > nuia, mia) e Fل⿳á- $\emptyset$ la sottoclasse nuova (ma cf. supra per bale e zale) nata in seguito alla massiccia entrata di prestiti da gr. e tc., in cui si sono poi integrati anche prestiti di altra origine, in part. fr. 
(44) aba, acadea, aga, alagea, alpaca ${ }^{1}, a_{1 p a c a^{2}}$, ana, anafora, andrea, angara, argea, baba, bacara ${ }^{1}$, bacara ${ }^{2}$, baclava, baga, bagea, balama, bale (pl), basma, beizadea, belea, besactea, bezea, bidinea (badana) ${ }^{15}$, bina, boccea, boia, brățea, bretea, bucea, cacealma, cafea, cafenea, caia, cana ${ }^{1}$ (canea), canale ${ }^{2}$ (pl), canapea, canara, canava, capama, caplama, capuchehaia, cara, caramea, cardama, carvasara, cașa, catifea, cazma, cătea, cealma, cecmegea, cercevea, chehaia, cherestea, cherhana, chiftea, chisea $^{1}$, chisea ${ }^{2}$, cișmea, citarea, ciulama, corăbiele (pl), cordea, croazea, cucuvea, cumbara, cupea, curama, curea, dairea (daira), dambla, dandana, danga, dara, debara, derea, diamanticale (pl), drepnea, dușumea, ecpaia, falbala, farfara, feregea, fermenea, fesfesele (pl), fidea, flanea, friganea, furda, geamparale (pl), gebea, gevrea, ghebea, ghilimele (pl), ghiulea, giubea, giurumea, giuvaiericale (pl), grădea, grimea, haimana, halca, halima, halva, haraba, harșa, hataia, havalea, hazna, iaca ${ }^{1}$, iavașa, imamea, iofca, ipingea, irmizea, istericale (pl), jaluzea, jartea, lalea, lamba, libadea, lichea, livrea, locma, lovele (pl), lulea, maca, macara, madea, mahala, mahmudea, maia, mala, malotea, manea, mangafa, manta, marda, masala, mascara, mărgea, măsea, menghinea, merdenea, mertepea, meterhanea, mia, micșunea, minarea, mola, mucava, muhaia, mumbaia, musaca, mușama, muștea, nacafa, narghilea, nevrica $(+\mathrm{pl})$, nicochera, nisfia (nisfea), novita, nuga, nuia, oca, oprea, orta, osanale (pl), pacea, pafta, paia, panama ${ }^{1}$, panama ${ }^{2}$, pangea, pansea, para, parcea, passa, patalama, patlagea, pavea, peltea, percea, perdea, peruzea, pijama, pingea, podea, politica $(+\mathrm{pl})$, preducea, proptea, protipenda, purcea, pușchea, pușlama, raia, rândunea, refenea, regea, rețea, rindea, rogodele (pl), rondea, rubia, saca, saftea, safterea, saia ${ }^{2}$, saia $^{3}$, saltea, sanda, saragea, sardea, sarma, sarsana, satara, schimbea, scordolea, sermaia (sermea), smicea, soarea, sofa, sofra, sosma, stea, stricnea, surcea, șabana, șahzadea, șale (pl), șandrama, șasla, șosea, șușanea ${ }^{1}$, șușanea ${ }^{2}$ tabla, tabulhana, tacla (+pl), tafta, taiga, tanana, tanea, tangara, tapangea, tara, tarama, tarapana, tarara, tarla, tasma, tava, tejghea, telelea, telemea, temenea, testea, teșcherea, tetrea, tinichea, trufanda, tura, turturea, tuslama, tutea, ulcea, ulema, urale (pl), vadea, vâlcea, vergea, vițea, vopsea, za (zea), zagara, zaharicale (pl), zaherea, zalhana, zarnacadea, zarpa, zăbrea, zeflemea, zenana, zi, zumaricale (pl), zurba.

In questa categoria rientrano 24 s. ereditati come tali dal lat. (andrea, bale (pl), bucea, cățea, curea, drepnea, mărgea, măsea, mia, nuia, purcea, pușchea, rândunea, rețea, smicea, stea, șale (pl), turturea, ulcea, vâlcea, vergea, vițea, za, zi), che rappresentano di fatto il nucleo originario dell'intera classe, e 1 creazione interna di natura onomatopeica (tarara). La maggior parte dei s. primitivi di

15 Tra parentesi tonde le var. secondarie appartenenti sempre a $F \sqrt{ } v_{-} \varnothing$ ma a una sottoclasse diversa da quella della var. principale. 
tipo $F \sqrt{ }{ }_{V}-\varnothing(254$, l'88,81\% di questa categoria e il 66,32\% del totale del corpus) sono tuttavia prestiti da varie lingue (in particolare tc., gr. e fr.).

Sempre in questa categoria ho incluso 2 retroformazioni da s. primitivi, F sg brățea (var. non corpus brăța, Florea 1984, 73) $\leftarrow$ F pl brăţal-e $\sim$ sg. brațal-ă, var. di brațar-ă < lat. BRACHIALE e F sg surcea $\leftarrow \mathrm{N}$ pl surcél-e $\sim$ sg surcél- $\emptyset<$ lat. *SURCĚLLU (cf. infra, §4.3.1), e 5 retroformazioni a partire da sg.GD/pl di s. der. (oprea, podea, proptea, stricnea, vopsea), in quanto nella risegmentazione dei sg.GD/pl originari e quindi nella creazione dei nuovi sg.NA ciò che viene meno è precisamente la coscienza della presenza del suff., il quale viene percepito in parte come elemento della radice e in parte come elemento del morfema di pl (cf. infra, §4.3.1).

4.2.1.1. Pseudoalterati. Una testimonianza lampante del "conflitto" tra etimologia e morfologia, per cui l'analisi dei parlanti entra in contrasto con i dati offerti dalla linguistica storica, è costituito dagli pseudoalterati, ovvero da s. storicamente primitivi percepiti per varie ragioni come contenenti il suff. dim. -ea, tale che si vengono a creare forme di sg parallele, con sostituzione dello pseudosuffisso /'ea/ con un suff. dim. vero e proprio (in part. -ică), ovvero forme di sg "primitive", retroformate con soppressione dello pseudosuffisso.

Da un lato, tanto in s. primitivi di eredità lat. $(8$, un terzo del totale) quanto in prestiti da altre lingue, il segmento sonoro finale /'êa/ è stato a volte percepito come suff. dim. -ea, tale che, in conformità con una tendenza ampiamente diffusa (cf. infra, §4.3.3), sono state create forme parallele di sg

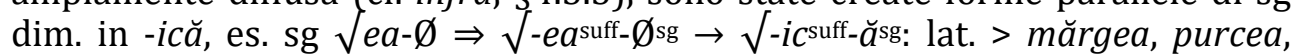
rândunea, surcea, turturea, ulcea, vâlcea, vițea $\rightarrow$ mărgică, purcică, rândunică, surcică, turturică, ulcică, vâlcică, vițică, tc. menekșe, patlıcan, pence > micșunea, pătlăgea, pingea $\rightarrow$ micșunică, pătlăgică, pingică ${ }^{16}$. L'associazione tra forme di sg.NA parallele /'êa/ $\approx$ /'ikə/ è talmente forte che si dà anche, seppur raramente, il fenomeno inverso: es. tc. pürçek $>$ percică $\rightarrow$ percea $^{17}$.

Dall'altro lato, sul modello di coppie alterato/primitivo quali uscătúrrad-eásuff-Øsg $\leftarrow$ uscătúrrad-ăsg, forme etimologicamente primitive

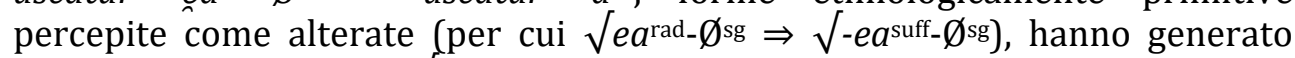
nuovi "primitivi" di tipo $\sqrt{-a ̆ s s: ~ e s . ~ t c . ~ m e n g e n e, ~ k e b e, ~ f r . ~ F ~ c r o i s e ́ e ~}>$ r. menghinea, ghebea, croazea $\rightarrow$ menghină, ghebă, croază (cf. anche Florea 1984, 76). Allo

16 Nel corpus tutte queste forme in -ică sono considerate var. secondarie, ad eccezione di rândunică, che compare come lemma indipendente senza rinvio a rândunea sia in DEX16 che in DOOM2, e di turturea, turturică, lemmi indipendenti in DEX16 ma lemmi plurivariante in DOOM2 (cf. anche infra).

17 E persino la sostituzione di м -el in forme dim. quali (м băiát- $\varnothing$, gíner-e $\rightarrow$ ) м giner-él, băineț-él $\rightarrow$ fam. giner-ícă, reg. băieț-ícă (Moroianu 2007, 689), che sono M $\sqrt{-a ̆}$. 
stesso modo in rogodele (pl), s. storicamente primitivo in ultima istanza di origine probabilmente slava ${ }^{18}$, il segmento /'ele/ ha potuto essere percepito come pl del suff. dim. -ea, tale che si è venuto a creare regressivamente un $\mathrm{s}$. "primitivo" rogoade (pl) 'id.' (non corpus, cf. MDA2): rogodélrad_e $e^{\mathrm{pl}} \Rightarrow$

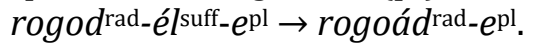

In maniera analoga, il fr. M pl guillemets, dapprima semiadattato nella forma guilemet (in E. Protopopescu, V. Popescu, Nou dicţionar portativ de toate zicerile radicale și streine reintroduse și introduse în limbă, cuprinzînd și termeni șciențifici și literari, București, 1862) e poi pienamente adattato come ghilmete (G. Ionescu, Călăuza tipografului, București, 1906), ha assunto infine - credo anche in virtù delle sonorità vicine a quelle di numerosi prestiti turchi - la forma ghilemele (accettata in DA, 1934, e SDLR, 1939) e quindi ghilimele (pl), oggi l'unica normativa (DOOM2). Che nonostante l'etimo questa parola sia percepita da almeno una parte dei parlanti come pl di un s. di tipo $F \sqrt{ } V_{\text {V́ }} \varnothing$ è dimostrato dalla nascita di sg.NA analogico ghilimea (già in Graur 1975, s.v.), la quale, ancorché non accettata dalla norma, appare utilizzata in $r$. attuale in contesti non solo colloquiali e scherzosi ma anche tecnici e professionali (es. istruzioni e tutorial redazionali e simili), tanto che - in linea con il fenomeno sopra descritto a proposito degli pseudoalterati - da essa si è persino venuta a creare la forma, per ora sporadica ed esclusivamente parodica e umoristica, ghilimică (per tutto questo, v. Zafiu 2016). Così anche corăbiele (pl) '(un tipo di) pasta secca', prestito dal tc. integrato in questa classe, ha formato, anche grazie al ravvicinamento paraetimologico a r. corabie 'barca' (< gr.), un sg.NA analogico corăbia /ko.rə'bia/ ${ }^{19}$.

4.2.2. Formati e composti. Dei restanti 83 s., la maggioranza quasi assoluta (76) sono suffissati, quasi tutti con il suff. -ea (75) (45.a), più 1 der. con

18 MDA2 distingue rocodele ${ }^{1} s f p$ var. di răcodelie $s f$ da rocodele ${ }^{2} s f p$ var. di rogodele $s f p$ (per la cui etimologia rinvia a un "răcodele", var. di răcodelie però non presente nel lemmario). Rogodele (pl) 'frutti piccoli di diverse sorte', ma anche 'erbe varie' (ȘDULR), è var. di un unico rocodele (pl), a sua volta var. pluralizzata (forse già per associazione con dim. F pl -ele) di sg răcodelie (roco-, ruco-, -deală, -dele) (pl. -i) 'lavoro manuale, oggetto fatto a mano' e, da qui, 'cucito, ricamo' (< sl. roqkodélije, bg. răkodélie). Secondo CDER, s.v. răcodelie, il passaggio di senso nelle forme rocodele, rogodele sarebbe dovuto a un ravvicinamento formale a $\mathrm{N}(\mathrm{F}) \mathrm{pl}$ roade 'frutti' ( $\sim \mathrm{sg} \mathrm{N}$ rod, rar. $\mathrm{F}$ roadă); personalmente propenderei per un slittamento di senso del termine, già pl, dai delicati motivi vegetali del ricamo tradizionale a erbe e frutti reali di piccole dimensioni.

19 MDA2, s.v. corăbea, considera questa forma una variante di corăbea $\leftarrow$ corabie + -ea, ritenendo il relativo pl un senso specifico di questa variante (giustificato nella definizione dal riferimento alla tradizionale forma "a barca"). Seguo invece DEX16 e DELR nel considerare corăbiele (pl) < tc. kurabiye, ant. ğurabiye 'id.' (< ar. ġurābìya; TETT 3, s.v. gurabiye), penetrato con il medesimo senso in tutte le lingue dei Balcani: cf. gr. kourampiés, bg. kurabíja, sb., mc. gurábija, alb. gurabíje, ar. curabé, gurabíe, gurbíe (Grămoșteni); (per queste forme cf. DDA). 
il suff. pl. -ale (45.b), mentre solo 7 sono i composti (45.c), di cui più della metà (baș-aga, beșli-aga, caimacam-aga, capudan-/căpitan-pașa), ancorché analizzabili in r. attuale (cf. baș-, beșliu, caimacam, căpitan e aga, pașa), sono di fatto stati presi a prestito come tali dal tc.:

(45) (a) adormițele (pl), albăstrea, alunea, bălticea, brândușea, brebenea, brumărea, bucățea, căpețea, cărticea, ciumărea, ciuturea, codănea, crengurea, crestățea, crușățea, duminecea, dungătea, fălcea, fântânea, fărâmele (pl), floricea, gălbenea, găurea, ghizdea, gogonea (+pl), gogoșea, îngerea, jucărea, lăcrimea, lingurea ${ }^{1}$, lingurea ${ }^{2}$, mărgărele (pl), măturea, mielărea, mielușea, minciunea, miorea, norea, ocheșele (pl), panglicea, părticea, păsărea, pătrățea, picătea, pielicea, plămânărea, plăsea, poezea, pogăcea, porumbea ${ }^{1}$, porumbea ${ }^{2}$, potecea, pupezea, rămurea, rocoteea, roșătea, rotoțele (pl), sălățea, sărățea, scândurea, secărea, străchinea, tâlhărea, telegea, uscățea, uscăturea, vâzdogea, ventricea, verigea, vindecea, vinețea, viorea, vișinea, zorea;

(b) cosmeticale (pl);

(c) automacara, baș-aga, beșli-aga, caimacam-aga, capudan-/căpitan-pașa, protostea, radiostea

Per quanto riguarda i derivati, cosmeticale ( $\mathrm{pl}$ ) fam. 'prodotti cosmetici', è formazione interna analizzabile sulla base di a., s. N cosmetic '(prodotto) cosmetico' + suff. pl (con valore collettivo) -ale (FCLR IV.1, 120; cf. anche DEX16, DELR, s.v. cosmetic; in MDA, stranamente, non compare). Includo questo termine in $\mathrm{F} \sqrt{\mathrm{V}}$ - $\varnothing$ in quanto - come suggeriscono anche la marca d'uso "fam." e persino l'assenza stessa di $\mathrm{sg}^{20}$ - pare formato per analogia con numerosi prestiti dal gr. storicamente integrati in questa classe, quali fam. istericale (pl), fam. nevricale $(+\mathrm{pl})$, fam. politicale $(+\mathrm{pl})$, zaharicale $(\mathrm{pl})$ ecc. ${ }^{21}$

20 Come si osserva in FCLR (IV.1, 120,124), diversi sg di s. di questo tipo lemmatizati in DA hanno pochissime (es. sg politica) o nessuna (es. sg nevrica) attestazioni testuali (in quest'ultimo caso essendo sospetti di essere formazioni lessicografiche $a d h o c$ ) ovvero sono in realtà prestiti non ancora adattati che conservano il senso pl dell'etimo gr. (es. zaharica). Nonostante questo, nel corpus ho rispettato l'indicazione delle fonti lessicografiche utilizzate (es. sg nevrica, come in DEX16 e in D0OM2).

${ }^{21}$ Una origine simile potrebbe avere anche chimicale (pl) pop. 'prodotti chimici (usati in casa o nell'industria alimentare)', per il quale DEX16, s.v. e DELR, s.v. chimic rimandano a td. pl Chemikalien, mentre MDA2, s.v. lo considera pl di F sg chimicală $\leftarrow$ a. chimic + -ală. Se questa ultima soluzione pare poco sostenibile in quanto sg $\mathrm{F}$ chimicală ha una sola attestazione e pare quindi piuttosto rifatto da chimicale (pl) (cf. FCLR IV.1, 120), non è invece escluso che l'eventuale prestito dal td. sia stato "attratto" in $\mathrm{F} \sqrt{\mathrm{V}}-\emptyset$ (v. anche fr. guillemets > ghilimele; cf. supra, §4.2.1.1), in part. per analogia con vari s. pl fam. e pop. in /i'kale/ con valore collettivo (es. cosmeticale, zaharicale). Nel dubbio, tuttavia, e sulla scorta della presenza del sg.NA in $\sqrt{a l a ̆, ~ n o n ~ h o ~ r i t e n u t o ~ o p p o r t u n o ~ i n c l u d e r e ~ c h i m i c a l e ~(p l) ~ n e l ~ c o r p u s . ~}$ 
Ma la quasi totalità dei s. non primitivi di tipo $\mathrm{F} \sqrt{\mathrm{V}}-\varnothing$ è costituita, di fatto, da formazioni interne di natura diminutivale con il suff. -ea (eventualmente nelle var. complesse -icea: bălticea, cărticea, floricea, părticea, pielicea, ventricea 22; -ărea: mielărea, forse brumărea, ciumărea ${ }^{23}$; -ușea: mielușea ${ }^{24}$; -urea: crengurea ${ }^{25}$ ), che costituiscono da sole quasi un quinto (per la precisione il 19,58\%) del totale del corpus.

4.2.2.1. Suffissati diminutivali. Essendo nella loro totalità formate con il suff. -ea o suoi composti26, tutti i 75 formati appartenenti a F $\sqrt{\hat{V}}$ - $\emptyset$ si inquadrano nella sottoclasse $\mathrm{F} \sqrt{e} a ́-\emptyset$, di cui costituiscono ben un terzo $(33,18 \%)$. Sebbene contraddistinto nel complesso dall'origine diminutivale, dal punto di vista morfo-semantico questo gruppo non è unitario, contenendo suffissati di varia natura ${ }^{27}$.

Oltre la metà di tali formazioni (45, pari al $60 \%$ dei suffissati diminutivali e all'11,75\% del corpus) sono diminutivi veri e propri, in cui l'aggiunta del suff. non modifica la classe lessico-semantica (criterio morfologico) e la formazione

22 I dim. in -icea (bălticea ecc.) possono essere considerati derivati tanto da X + -icea quanto da $\mathrm{X}$-icică con sostituzione di suff. -(ic)ică $\rightarrow-(i c) e a$; in DEX16 sono considerati derivati quando (erroneamente) da X + -ea (bălticea, cărticea) quando da X + -icea (floricea, părticea), oppure da X-icică con sostituzione di suff. (pielicea); per ventricea, per cui DEX16 dà "etimologia sconosciuta", adotto qui la soluzione di MDA2: $\leftarrow$ F vintre + -icea.

23 Il segmento /ə'rea/ compare spesso in nomi di fiori e pianticelle passibili di essere derivati, ma la sua natura di suffisso non è sempre certa: per quanto riguarda i s. del corpus, mielărea 'agnocasto (Vitex agnus-castus)' è considerata $\leftarrow$ miel 'agnello' + -ărea tanto in DEX16 quanto in MDA2, mentre per quanto riguarda l'origine di brumărea 'flogo (Phlox drummondii; Phlox paniculata)', ciumărea 'capraggine (Galega officinalis)' e plămânărea 'polmonaria (Pulmonaria officinalis)' i pareri sono divisi, cf. Infra, n. 40.

24 Considerato F di m mielușel; anche in questo caso sono possibili diverse analisi: miel + -uş-el (cf. DEX16), oppure miel-us + -el (cf. MDA2).

25 DEX16: creangă + -urea, MDA2: pl crenguri + -ea.

${ }^{26}$ Purtroppo il volume del trattato accademico Formarea cuvintelor în limba română che tratta la suffissazione denominale (FCLR IV.1) arriva solo fino alla lettera C, e altri studi d'insieme - a partire dal classico Pascu (1916) fino a Coteanu, Bidu-Vrănceanu, Forăscu $(1985,188-224$; cf. anche Coteanu 2007) e Vasiliu (2001) - o studi specifici anche recenti che trattano il suff. -ea (es. Şerban 2009 e 2010) non toccano che aspetti generali e generici. In attesa di uno studio monografico dedicato al suff. -ea in un futuro vol. di FCLR, il quadro qui tracciato ha, naturalmente, carattere parziale e di ipotesi di lavoro.

27 In quanto segue mi ispiro alla distinzione di Moroianu (2015, 304-305) tra (a) dim. reali (formali e semantici), (b) dim. esclusivamente formali, "il cui significato principale non è diminutivo, ma la cui forma è percepita con questo valore" e (c) falsi diminutivi, "che non possono più essere considerati tali, nonostante la forma": (a) corrisponde a quelli che chiamo semplimente "diminutivi veri e propri", mentre considero (b) e (c) insieme come "diminutivi apparenti". 
risultante non implica un cambiamento di senso della base ma una diminuzione o una connotazione affettiva (criterio semantico) ${ }^{28}$; data la sua natura, questo primo sottoinsieme è costituito esclusivamente da desostantivali:

(46) bălticea $(\leftarrow \mathrm{F}$ baltă $)$, brândușea $(\leftarrow \mathrm{F}$ baltă $)$, brebenea $(\leftarrow \mathrm{M}$ breabăn $)$, bucățea $(\leftarrow \mathrm{F}$ bucată $)$, căpețea $(\leftarrow \mathrm{N} * \text { căpeț })^{29}$, cărticea $(\leftarrow \mathrm{F}$ carte $)$, ciuturea $(\leftarrow \mathrm{F}$ ciutură), codănea $(\leftarrow \mathrm{M}, \mathrm{F}$ codan, $-\breve{a})$, crengurea $(\leftarrow \mathrm{F}$ creangă), fălcea $(\leftarrow \mathrm{F}$ falcă), fărâmele $(\mathrm{pl})(\leftarrow \mathrm{F}$ fărâmă), fântânea $(\leftarrow \mathrm{F}$ fântână), floricea $(\leftarrow \mathrm{F}$ floarea $)$, ghizdea $(\leftarrow \mathrm{N}$ ghizd), gogoșea $(\leftarrow \mathrm{M}$ gogoș $)$, jucărea $(\leftarrow \mathrm{F}$ jucărie $)$, lăcrimea $\left(\leftarrow \mathrm{F}\right.$ lacrimă), lingurea ${ }^{1} \leftarrow \mathrm{s}$. lingură, lingurea ${ }^{2} \leftarrow \mathrm{s}$. lingură, mărgărele $(\mathrm{pl})^{30}(\leftarrow \mathrm{F}$ mărgea $\times \mathrm{N}$, $\mathrm{M}$ mărgăritar $)$, mielușea $\left(\leftarrow \mathrm{M}\right.$ miel), minciunea $(\leftarrow \mathrm{F} \text { minciună })^{31}$, miorea $(\leftarrow \mathrm{F}$ mioară $)$, panglicea $(\leftarrow \mathrm{F}$ panglică $)$, părticea $(\leftarrow \mathrm{F}$ parte), păsărea $(\leftarrow \mathrm{F}$ pasăre $)$, pătrățea $(\leftarrow \mathrm{N}$ pătrat $)$, pielicea $(\leftarrow \mathrm{F}$ piele $)$, plăsea $(\leftarrow \mathrm{F}$ plasă $)$, poezea $(\leftarrow \mathrm{F}$ poezie $)$, pogăcea $(\leftarrow \mathrm{F}$ pogace $)$, porumbea ${ }^{1} \leftarrow \mathrm{s}$. porumb $\breve{a ̆}^{32}$, porumbea ${ }^{2} \leftarrow$ s. porumb, $-\breve{a ̆}^{33}$, potecea $(\leftarrow \mathrm{F}$ potecă), pupezea $(\leftarrow \mathrm{F}$ pupăză), rămurea $(\leftarrow$ $\mathrm{F}$ ramură), sălățea $(\leftarrow \mathrm{F}$ salată), scândurea $(\leftarrow \mathrm{F}$ scândură), străchinea $(\leftarrow$ F strachină), telegea ( $\leftarrow$ F teleagă), uscăturea $(\leftarrow \mathrm{F}$ uscătură), vâzdogea $(\leftarrow$ $\mathrm{F}$ vâzdoagă $)$, verigea $(\leftarrow \mathrm{F}$ verigă $)$, viorea $(\leftarrow \mathrm{F}$ vioară $)$, vișinea $(\leftarrow \mathrm{F}$ vișină $)$.

${ }^{28}$ Ho incluso in questo gruppo anche casi in cui nella lingua standard contemporanea il legame sematico è venuto allentandosi, gli alterati essendosi più o meno lessicalizzati: es. plăsea 'rivestimento del manico di coltelli e sim.' $\leftarrow$ plasă ${ }^{1}$ 'rete', ma anche (rar.) "III. Limbă de cuțit; tăiș” (DEX16, s.v.), "34 [ant. e rar.] Lamă de metal. 35 [reg.] Tăiș de cuțit. 36 [ant. e reg.] Mâner al unui cuțit" (MDA2, s.v.), o căpețea (cf. n. successiva).

29 Sulla scorta di PEW (277) e DELR (s.v. căpețea1) considero căpețea 'cavezza' ("parte a frâului alcătuită din curelele care trec peste capul și botul calului și ale cărei capete inferioare sunt prinse de inelele zăbalei"; DEX16), come rifatto da N pl căpețel-e sg căpețel 'id.' $\leftarrow$ N sg *căpet $\sim \mathrm{pl}$ *căpețe $(<\mathrm{N}$ sg CAPĬTIU $\sim \mathrm{pl} \mathrm{CAPITTIA,} \mathrm{ben} \mathrm{attestato} \mathrm{a} \mathrm{livello} \mathrm{romanzo,} \mathrm{cf.} \mathrm{it.} \mathrm{cavezza,} \mathrm{fr.}$ ant. chevece ecc.; REW 1637) + -el.

30 Di etimo incerto, ma che pare difficile non considerare come dim. sorto dalla contaminazione di F pl mărgele 'perl(in)e' (sg mărgea < lat. MARGĔLLA) con N e M mărgăritar (< gr. margaritári), N e M mărgărit (< gr. margarítēs), entrambi 'perla' e 'mughetto', anche tramite forme diminutivali quali N mărgăritărel, F mărgăluță, mărgălușă, in cui si intrecciano il senso 'perl(in)a' e le denominazioni di alcune piante dai piccoli fiori bianchi, come il mughetto (Lithospermum arvense) e l'erba perla minore o bianca (Convallaria majalis). Per il carattere chiaramente diminutivale lo inserisco tra gli alterati.

31 Poiché minciună è attestato sia con il senso 'bugia (menzogna)' sia con quello 'bugia (dolce)', minciunea 'piccola bugia (menzogna)' e (soprattutto al pl) 'bugie (dolci)' può essere considerato alterato.

32 Si tratta di s. F porumbă ${ }^{1}$ 'frutto del pruno selvatico spinoso (Prunus spinosa)' < lat. PALUMBA (DEX16) / *POLUMBA (MDA2).

33 Ovvero s. M,F porumb, -ă 'colombo; piccione' < lat. PALUMBU. 
Un secondo sottoinsieme di suffissati è costituito dai diminutivi apparenti (18 s.), ovvero da suffissati tanto denominali ( $8 \mathrm{~s}$.) quanto deaggettivali $(10 \mathrm{~s}$.) in cui il formato è formalmente un dim. ma semanticamente non ha senso alterato ma piuttosto derivato, esprimendo in genere il possesso di una certa caratteristica o un qualche tipo di associazione legata alla base; questo tipo di formazione è piuttosto frequente nella creazione di nomi di erbacee a portamento nano che producono fiori, qui precedute da "+":

(47) (a) +adormițele (pl) ( $\leftarrow$ a. adormit), +albăstrea $(\leftarrow$ a. albastru), ${ }^{+}$crestățea $(\leftarrow$ a. crestat $),{ }^{+}$cruşățea $(\leftarrow$ a. crușit $),{ }^{+}$dungățea $(\leftarrow$ a. dungat $)$, + gălbenea $(\leftarrow$ a. galben $),{ }^{+}$ocheșele $(\mathrm{pl})(\leftarrow$ a. oacheș $)$, picățea $(\leftarrow$ a. picat $)$, sărățea $(\leftarrow$ a. sărat $)$, uscățea $(\leftarrow$ a. uscat $)$, ${ }^{+}$vinețea $(\leftarrow \text { a. vânăt })^{34}$;

(b) + duminecea $(\leftarrow \mathrm{s}$. F duminecă $),+$ îngerea $(\leftarrow \mathrm{s}$. M înger $),+$ mielărea $(\leftarrow \mathrm{s}$. $\mathrm{M}$ miel $)^{35},{ }^{+}$norea $(\leftarrow \mathrm{s} . \mathrm{M} \text { nor })^{36},+$ rototele $(\mathrm{pl})(\leftarrow \mathrm{s} \text {. F roată })^{37},{ }^{+}$secărea $(\leftarrow$ S. F secară), ${ }^{+}$tâlhărea $(\leftarrow \mathrm{s}$. M tâlhar $),{ }^{+}$ventricea $(\leftarrow \mathrm{s} \text {. F vintre })^{38},{ }^{+}$zorea $(\leftarrow$ s. F/M zori $(\mathrm{pl}))$.

Naturalmente, il confine tra i due tipi sopra descritti, così come tra diminutivi e formati lessicalizzati, è assai fluido, vuoi perché numerose forme alterate si sono spesso anche lessicalizzate con sensi specifici ${ }^{39}$, vuoi perché

34 In linea teorica le forme deaggettivali possono essere spiegate come lessicalizzazioni del $\mathrm{F}$ dell'alterato dell'aggettivo (cf. a. albăstrel $\leftarrow$ a. albastru, a. ocheșel $\leftarrow$ a. oacheș, a. sărățel $\leftarrow$ a. sărat, a. uscățel $\leftarrow$ a. uscat, a. vinețel $\leftarrow$ a. vânăt) o come "mozione di genere" s. $\mathrm{M} \rightarrow \mathrm{s}$. $\mathrm{F}$ (cf. m picățel, $\mathrm{M}$ crestătel con senso id. al F; cf. anche le coppie di alterati desostantivali con senso id. M brebenel / F brebenea, N pătrățel / F pătrătea); questa spiegazione, tuttavia, non è sempre possibile in quanto a. alterato o s. M con senso vicino non sono sempre attestati. Casi quali adormițele (pl), crușățea, gălbenea o dungățea, ma molto probabilmente anche altri in cui pure è attestato l'a. o un $\mathrm{s}$. M alterati, si possono spiegare come derivazione diretta con un suff. sostantivale desostantivale e deaggettivale inerentemente $\mathrm{F}$ sg.NA -ea $\sim \mathrm{sg}$.GD/pl -ele, part. frequente nella formazione di nomi di pianticelle e fiori (la prevalenza del genere $\mathrm{F}$ e del $\mathrm{pl}$ in questo tipo di formati è prob. dettata dall'analogia con iperonimi quali F sg iarbă, F pl flori).

35 Cf. n. 24.

36 DEX16 e MDA2 danno "etimologia sconosciuta"; seguo qui la proposta di SDLR.

37 Così DEX16, MDA2, CDER, ma la derivazione pare non diretta e con l'intervento di fenomeni analogici (cf. anche roșățea).

38 Cf. n. 22.

39 Ad es. alunea 'nocciolina' e, per est., 'lentiggine; neo' è dim. di alună 'nocciola', ma anche nome di una danza popolare e, al pl alunele, il nome di alcune piante, così come măturea 'scopetta; spazzolina' è dim. di mătură 'scopa', ma al pl măturele indica la 'Centaurea rutifolia subsp. jurineifolia' (presente solo in Romania e Bulgaria; non ho trovato un traducente it.) e al pl art. măturelele una danza popolare e la relativa melodia, e găurea 'buchetto, forellino' è dim. di gaură 'buco, foro' ma indica anche, al pl găurele, un tipo di ricamo su tela in cui si estraggono alcuni fili qua e là. Similmente, gogonea è formalmente dim. di obs. e rar. M gogón 'oggetto piccolo, rotondo e duro' (cf. MDA2, s.v. gogon), ma in r. standard compare soprattutto al pl con il senso di 'pomodoro acerbo che si mette sottaceto' (cf. MDA2, s.v. gogonea). 
l'etimologia di una parola non è sempre chiara, tale che spesso - laddove non vi sia consenso e un'ipotesi non paia più verosimile di altre - non è possibile stabilire un quadro univoco del processo morfosemantico che ha portato alla formazione in questione né, quindi, della sua struttura morfologica ${ }^{40}$. Un piccolo sottoinsieme di suffissati in -ea $(10$ s., il $13,33 \%$ dei suffissati e il $2,61 \%$ del corpus) è infatti costituito da formati non facilmente inquadrabili nella casistica esemplare precedente:

$(48){ }^{(+)}$alunea $\left(\leftarrow\right.$ F alună), ${ }^{+}$brumărea $\left(\leftarrow \mathrm{F}\right.$ brumă, a. brumărel?), ${ }^{+}$ciumărea $(\leftarrow \mathrm{F}$ ciumă, M ciumar?), găurea $(\leftarrow \mathrm{F}$ gaură), gogonea $(+\mathrm{pl})(\leftarrow \mathrm{M}$ gogon $)$, ${ }^{+}+$măturea $(\leftarrow \mathrm{F}$ mătură $),{ }^{+}$plămânărea $(\leftarrow \mathrm{F}$ alună $),{ }^{+}$rocoțea $(\leftarrow \mathrm{F}$ rocoină? $)$, +roșătea $\left(\leftarrow \mathrm{F}\right.$ roșeață, a. roșu?), ${ }^{+}$vindecea $(\leftarrow \mathrm{v}$ vindeca, $\mathrm{F}$ vindecă? $)$.

In particolare per i numerosi nomi di piante e fiori presenti in questi ultimi due sottoinsiemi una discussione etimologica approfondita (che non rientra negli obiettivi immediati della presente serie di studi) dovrebbe tenere conto delle numerose varianti, della circolazione, delle forme regionali, del frequente intervento dell'analogia tra parole e tra piante, e persino della confusione tra queste ultime.

40 Ad es. brumărea può essere considerato formato come der. desostantivale $\leftarrow$ F brumă + -ărea (DEX16; DELR, s.v. brumă, non precisa) (tipo 47.b) ovvero come lessicalizzazione del F dell'a. brumărel con senso specifico 'flogo' (tipo 47.a); ciumărea 'capraggine (Galega officinalis)' può essere der. da F ciumă 'peste' + -ărea (DEX16) (tipo 47.b) ovvero da M ciumar 'stramonio (Datura stramonium)' + -ea (MDA2; DELR, s.v. ciumă) (tipo 47.a), ma anche essere considerato alterato di M ciumar 'capraggine' (MDA2, s.v. ciumar) (tipo 46), sempre che con questo ultimo senso ciumar non sia di fatto un primitivo rifatto da ciumărea, percepito come alterato; plămânărea 'polmonaria (Pulmonaria officinalis)' può essere dim. di F plămânare 'id.' (MDA2, s.v.; a sua volta da M plămân 'polmone' + -are, MDA2, s.v.) (tipo 46), ma anche sg analogico da F pl plămânărele sg plămânărică o sostituzione di suffisso da sg plămânărică $\rightarrow$ plămânărea $\mathrm{e}$ quindi der. di plămân 'polmone' + -ărică (DEX16, s.v. plămânărică) (tipo 47.b). Allo stesso modo, roșătea 'giunco fiorito (Butomus umbellatus)' può essere der. da s. roșeață 'rossore' (SDLR) (tipo 47.b), ma anche essere formazione analogica da a. roșu 'rosso' (DEX16, MDA2) (tipo 47.b). Vindecea 'erba betonica (Betonica/Stachys officinalis)' è in genere considerato der. da v. vindeca 'guarire' + -ea (DEX16, MDA2, CDER, s.v. vindeca), ma sarebbe l'unico caso di der. deverbale presente nel corpus (escludo qui l'ipotesi stricnea $\leftarrow$ stricni + -ea di DEX16; cf. infra, n. 48); in considerazione del fatto che la suffissazione denominale con -ea è particolarmente frequente nella formazione di nomi di piante, tuttavia, potrebbe non essere errato vedere in vindecea un dim. di s. F reg. vindécă 'id.' (a sua volta derivato a suff. zero di v. vindeca) (tipo 46), sempre che quest'ultimo non sia a sua volta un sg rifatto proprio da vindecea o da vindecuţă 'id.'. Nel caso di rocotea 'centocchio gramignola (Stellaria graminea)', invece, avremmo a che fare con una formazione analogica da F rocoină, var. di F răcovină (et. sc., DEX16, MDA2; < sl. [sb., rs.] rakovina, CDER, s.v. rac), che tra i vari sensi ha anche quello di Stellaria graminea, con un chiaro carattere di forma diminutivale, ma non così linearmente interpretabile. 
4.3. Varianti morfologiche. Numerosi s. appartenenti ad F $\sqrt{\mathrm{V}}-\emptyset$ presentano una o più varianti morfologiche (42 s.), appartenenti sia ad altri sottotipi della stessa classe $\left(6 \mathrm{~s}\right.$.) sia ad altre classi flessive $(39 \mathrm{~s} \text {. })^{41}$, in part. $\mathrm{F} \sqrt{-a} \breve{a}, \mathrm{~F} \sqrt{-e}$ e $\mathrm{M} \sqrt{-} \breve{a}$, ovvero le classi estremamente numerose dei F con sg.NA in - $\breve{a}$ e in - $e$ e quella estremamente ridotta dei $\mathrm{M}$ con sg in - $\breve{a}$.

Per quanto riguarda il corpus lessicografico analizzato, da un lato, un buon numero di termini $(25 \mathrm{~s}$.) presenta var. morfologiche secondarie appartenenti ad altre classi flessive (per le "varianti" in -ică, cf. infra, §4.3.2) ) $^{42}$ :

(49) (a) $\sqrt{a}-\emptyset \approx \sqrt{a ́ l-a ̆: ~ n a c a f a / n a ̆ c a ̆ f a l a ̆, ~ s a n d a ~<~ s a n d a l a ̆, ~ s a r m a ~<~ s a r m a l a ̆, ~ z a ~}$

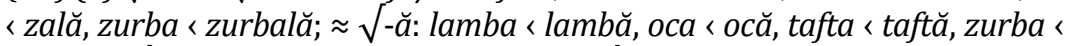
zurbă; $\approx \sqrt{ }$-íe: angara < angarie, angărie; $\approx \sqrt{a ́ i}$-e: trandada < trandadaie;

(b) $\sqrt{e a ́}-\emptyset \approx \sqrt{a ́ a}-e:$ cucuvea $<$ cucuvaie; $\approx \sqrt{ }-\breve{a}$ : croazea $<$ croază $\approx \sqrt{e}$ é $\varnothing$ : minarea <minare $43 ; \approx \sqrt{-e a ́ l-a ̆: ~ v o p s e a ~}<$ vopseală, podea $<$ podeală, stricnea 〈 stricneală; $\approx \sqrt{e ́ l}$ l-ă: cordea < cordelă, grădea > grădelă; $\approx \sqrt{i}$-e: citarea < citarie; $\approx \sqrt{\text { eáu }}$-ă: stea < steauă;

(c) $\sqrt{i a ́}-\emptyset \approx \sqrt{a ́ i}$-e: capuchehaia/capuchehaie, chehaia/capuchehaie; $\approx$ $\sqrt{i a ́ l}-$ ă: mia < mială $; \approx \sqrt{ }-$ ie: nisfia $<$ nesfie ${ }^{44}$

(d) $\sqrt{i}-\varnothing \approx \sqrt{i} \dot{i}-a ̆$ : $z i<$ <iuă.

Dall'altro, alcuni dei vocaboli di tipo $\mathrm{F} \sqrt{\mathrm{V}-} \emptyset$ inventariati nel corpus $(13$ s.) sono essi stessi var. secondarie di lemmi appartenenti ad altre classi flessive (soprattutto $\mathrm{F} \sqrt{-a}$ ); di queste, solo aga e pașa sono registrate in DOOM2 come lemmi a sé stanti45:

41 La differenza tra la somma delle due categorie e il totale è dovuta al fatto che za, zurba, nisfia hanno nel corpus più varianti: zală e zea, zurbă e zurbală, nesfie e nisfea (cf. infra).

42 In quanto segue, "/" tra forme indica che in DOOM2 entrambe le varianti sono a lemma e "" separa la var. considera primaria da quella secondaria secondo DEX16; il grassetto segnala la variante considerata principale in DEX16 e/o messa al primo posto in D0OM2. Gli inventari hanno la seguente

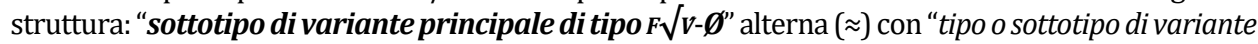
$n r$. 1": variante principale < variante secondaria; alterna con "tipo o sottotipo di variante $n r .2$ ": variante principale < variante secondaria ecc. Ad es. (49.a) indica che per i s. Fل⿳á- $\emptyset$ nacafa, sanda, sarma, za, zurba il corpus registra varianti secondarie di tipo $\mathrm{F} \sqrt{-a ̆ ~ i l ~ c u i ~ t e m a ~ t e r m i n a ~ i n ~[' a l] ~(~} \sqrt{a} l-a ̆)$ : năcăfală, sandală, sarmală, zală, zurbală, mentre (50.a) indica che per i s. Mل-ă agă, beșleagă, pașă e $\mathrm{F} \sqrt{ }$-ă protipendadă, tară sono presenti le var. F $\sqrt{a}-\emptyset$ aga, beșli-aga, pașa, protipenda, tara.

43 La forma obs. minare /mina're/ è l'unico caso presente nel corpus di prestito non adattato dal tc., caso non raro tra gli hapax attesati a cavallo tra XVIII e XIX sec. (Suciu 2013, cap. 4); caso analogo, non presente nel corpus, è ad es. il s e a. obs. pembe /pem'be/ < pembea (MDA2, s.v.).

44 Per comodità di reperimento seguo qui l'uso corrente di trattare i s. di tipo $\sqrt{i}$ í́- $\varnothing$ come gruppo a parte (ma cf. supra, §4.1.3).

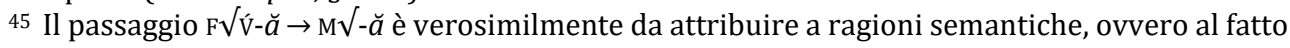
che tali s. designa(va)no persone di sesso maschile, sul modello di m sg tat-ă 'padre; papa' (cf. Diaconescu 1970, 105; CDER, s.v. agă). 
(50) (a) F,M $\sqrt{-a ̆} \approx \mathrm{F} \sqrt{a ́}-\varnothing:$ M agă < aga, M beșleagă < beșli-aga, protipendadă < protipenda, м pașă < pașa, tară < tara; $\approx \sqrt{\text { ená- } \emptyset: ~ m e n g h i n a ̆ ~}<$ menghinea, minghinea, ghebă < ghebea;

(b) $\sqrt{-e a ́ l-a ̆ ~} \approx \sqrt{e}$ é- $\emptyset$ : opreală $<$ oprea;

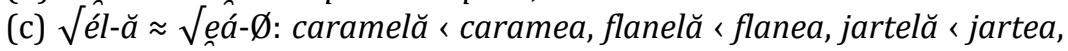
rondelă $<$ rondea;

(d) $\sqrt{e}-\underline{u} \approx \sqrt{e}$ é-Ø: $\mathrm{N}$ cupeu < cupea.

Infine, è presente anche un certo grado di variazione interna (6 s.) tra sottotipi di $\mathrm{F} \sqrt{\mathrm{V}}-\varnothing$ :

(51) (a) $\sqrt{a}-\varnothing \approx \sqrt{e} a ́-\varnothing:$ cana $<$ canea, za $<$ zea;

(b) $\sqrt{e a ́}-\emptyset \approx \sqrt{a} a-\emptyset:$ bidinea $<$ badana, dairea $<$ daira;

(c) $\sqrt{i} \hat{i} a ́-\emptyset \approx \sqrt{e} a-\emptyset:$ nisfia $<$ nisfea; sermaia $<$ sermea.

Per quanto riguarda l'origine delle diverse var., un certo numero di casi di variazione tra $\mathrm{F} \sqrt{\mathrm{V}}-\varnothing$ e $\mathrm{F} \sqrt{ }-\breve{a}$, es. menghinea > menghină, è certamente dovuto alla rianalisi della sequenza finale /'ea/ come elemento diminutivale (cf. supra quanto detto a proposito degli pseudoalterati, §4.2.1.1), mentre altri sono possibili doppietti etimologici, es. gr. angareía > angara e angarie (angă-), tc. nısfiye > nisfia, nisfea e nesfie, tc. çetare (çi-) > citarea e tc. çetari > citarie (cf. anche $\mathrm{N}$ citariu, in MDA2), forse dovuti alla penetrazione di uno stesso termine per vie differenti (es. scritto, colto vs parlato, popolare) e/o in momenti diversi, mentre altri ancora rappresentano verosimilmente il risultato di prestiti con filiere multiple, es. tafta < tc. tafta, fr. taffetas, gr. taftás vs taftă < rus., pol. tafta ${ }^{46}$.

4.3.1. Retroformazioni. Nel quadro complessivo della variabilità morfologica interclasse (49-50) è interessante rilevare l'esistenza di alcuni pattern specifici ricorrenti, in cui si inquadra quasi la metà (20 elementi, il $5,22 \%$ del corpus) dei s. che presentano var. morfologiche:

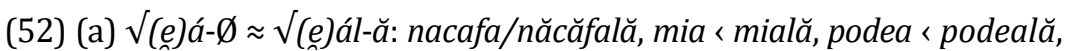
sanda < sandală, sarma < sarmală, stricnea <stricneală, vopsea < vopseală, $z a<z a l a ̆, z u r b a<z u r b a l a ̆ ~(F \sqrt{\mathrm{V}}-\varnothing$ principale); oprea > opreală $(\mathrm{F} \sqrt{\mathrm{V}}-\varnothing$ secondaria);

\footnotetext{
46 Per il resto, protipenda potrebbe essere l'esito fonetico, tramite parlato, dell'etimo gr. prốtē pentáda /,proti pen'da $\delta a /$, con /'da $\delta a / ~ \rightarrow / \mathrm{da} /$ per dileguo di / $\delta /$ intervocalico, ovvero il risultato dell'inquadramento fonomorfologico di questo prestito in $\mathrm{F} \sqrt{\mathrm{V}}-\varnothing$, vuoi direttamente vuoi tramite la forma etimologica protipendadă, motivato dall'alto numero di grecismi contenuti in questa classe, mentre $\mathrm{F}$ cupea pare essere il risultato primario dell'adattamento su base fonomorfologica di fr. $\mathrm{M}$ coupé (cf. anche fr. M pavé > r. F pavea), poi passato a N cupeu per influenza del genere dell'originale (cf. infra).
} 
(b) $\sqrt{e}$ á- $\emptyset \approx \sqrt{e ́ l}-$ ă: caramea > caramelă, flanea > flanelă, jartea > jartelă, rondea > rondelă ( $\mathrm{F} \sqrt{\mathrm{V}}-\varnothing$ secondaria); cordea < cordelă, grădea < grădelă ( $\mathrm{F} \sqrt{\mathrm{V}}-\varnothing$ principale);

(c) $\sqrt{a}-\emptyset \approx \sqrt{a ́ i}-e:$ capuchehaia/capuchehaie, chehaia/capuchehaie, cucuvea < cucuvaie; trandada < trandadaie ( $\mathrm{F} \sqrt{\mathrm{v}}-\varnothing$ principale).

L'esistenza delle var. in (52.a) e (52.b) è dovuta a processi di retroformazione - con conseguente cambio di classe flessiva - causati dall'omofonia tra le forme di sg.GD/pl dei s. appartenenti a $F \sqrt{ } \hat{V}-\emptyset$ e quelle di alcuni tipi di s. $\mathrm{F} \sqrt{-a ̆}$; nella fattispecie:

(i) sg.GD/pl $\sqrt{e}-l e(\sqrt{i e ́}-l e)$, pop. $\sqrt{e}-l i(\sqrt{i e ́}-l i)(\mathrm{cf}$. infra, §4.3.1) del

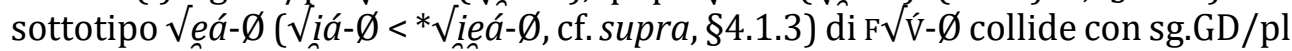
obs. e pop. -él-e, lett. -él-i di formati con il suff. sostantivale deverbale sg.NA -eál-ă $(\sqrt{i}$-ál-ă $<* \sqrt{i}$-ęál-ă, cf. supra, §4.1.3) (<sl.);

(ii) sg.GD/pl $\sqrt{e}-l e$ e $\sqrt{a}-l e$, pop. $\sqrt{e}-l i, \sqrt{a}-l i(\mathrm{cf}$. infra, $\S 4$ 4.3.1) dei sottotipi

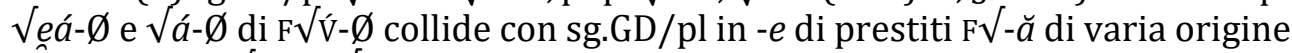
con radice in $\sqrt{e} l-\mathrm{o} \sqrt{a} l$ -

A causa di tale collisione, in (i) il segmento /'ele/ (/'elì /) -élsuff-e $e^{\text {pl }}$ $\left(-e^{\text {suff- }} i^{\mathrm{pl}}\right)$ viene rianalizzato come $\sqrt{e^{\text {rad }}-l e^{\mathrm{pl}}\left(-e^{\text {suff-l}}-l p^{\mathrm{pl}}\right)}$, es. v. opri $\rightarrow$ s. sg.NA

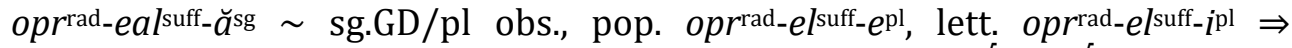

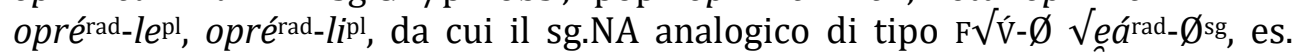

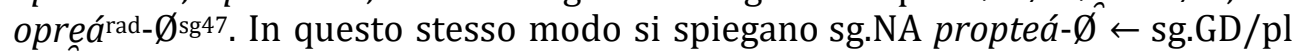

47 Un'analisi alternativa potrebbe essere che /'ele/ (/'eli $/$ /) sia interpretato come suff. dim. sg.GD/pl - ésuff-lepl (-ésuff-lipl) e che quindi il sg.NA analogico sia analizzabile come opr rad-éásuff-Øss. Contro questa analisi depone il fatto che per i s. in questione forme di sg.NA parallele in -ică (es. * oprică, ${ }^{*}$ podică ecc.), invece estremamente frequenti nei s. alterati e pseudoalterati in /'ea/ (cf. infra, §4.3.2) paiono assenti, per i s. in questione non essendo registrate né nelle opere lessicografiche utilizzate per la creazione del corpus di s. $F \sqrt{V}-\varnothing$ né in MDA2. Nel ricco inventario di forme reg. e pop. censite da I.A. Florea sulla base di atlanti linguistici e raccolte di folklore parrebbe comparire un'unica forma di questo tipo, suppostamente attestata da NALR Ban., domanda 460, punto 25, che l'autore letterarizza "amețíce" 'vertigini, stordimento' e considera pl di un sg "amețấcă" (ovvero lett. *amețică con pronuncia reg. /tsi/ per lett. /tsi/ «ți) der. da sg amețea 'id.' $\leftarrow$ pl amețeli $\sim$ sg amețeală 'id.' $\leftarrow$ v. ameți + -eală (Florea 1984, 75). Tuttavia, una verifica nello schedario di NALR Ban., dom. 460 (Când îți vine rău și ți se face negru în fața ochilor, de parcă se învârte pământul cu tine, ce zici că ai?), pt. 25 (Luncavița) rivela che le forme effettivamente notate dal raccoglitore Eugen Beltechi sono le seguenti: "amięțî́t, -țíćé, [k] amiěțálă; -țăli" (dove [k] sta per "corretto dall'informatore con") (cf. NALR-B VI.1, 108; ringrazio qui il collega Nicolae Mocanu dell'Istituto di Linguistica e Storia letteraria "Sextil Pușcariu" dell'Accademia di Romania, Filiale Cluj-Napoca, per l'accesso al volume in corso di stampa). La forma in questione è quindi "amiěțíće" , con 'ć, che nella trascrizione NALR indica l'affricata alveopalatale sorda reg. /t6/, perlopiû corrispondente a lett. /t/ davanti a vocale anteriore (es. reg. fraće, frunće vs lett. frate, frunte), da letterarizzare quindi "amețíte" (lett. amețíte), la quale non è F pl di un fantomatico reg. F sg *amețîcă $\leftarrow$ amețea bensì $\mathrm{N}$ pl di reg. amețít (lett. amețít), part. pass. sostantivato di v. ameți, attestato col senso 'vertigine, stordimento', ma con pl -uri, in Artur Gorovei, 
propté-le $\Leftarrow$ propt-él-e $\sim$ sg.NA propt-eal-ă $(\leftarrow$ v. propti $)$ e le coppie di var. stricnea $\approx$ stricneală $(\leftarrow$ v. stricni $)$, podea $\approx$ podeală $(\leftarrow$ v. podi $)$, vopsea $\approx$ vopseală $(\leftarrow \text { v. vopsi })^{48}$. In simili casi di variabilità morfologica, nella lingua standard - e quindi nel campione rappresentativo qui esaminato - è stata selezionata a volte la sola var. $\sqrt{e} e-\emptyset$ a volte la sola var. $\sqrt{ }$-eal-ă oppure, come in DEX16 per i casi qui esaminati, entrambe.

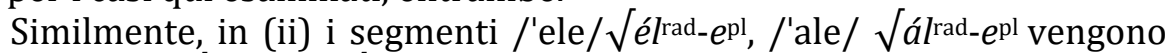

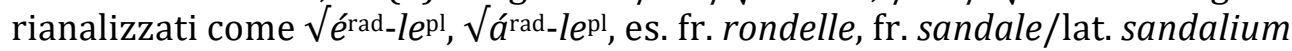

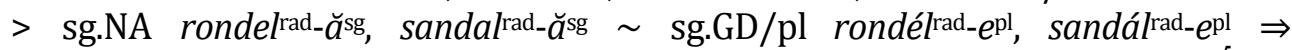
rondérad-lepl, sandárad_lepl, donde la nascita di sg.NA analogici di tipo $\mathrm{F} \sqrt{\mathrm{V}}-\varnothing$ :

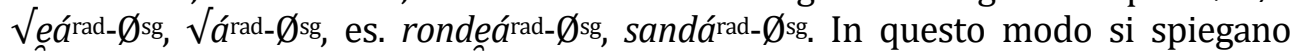
forme quali reg. sg.NA brățea 'bracciale' rispetto a brățară (< lat. BRACHIALE), tramite pl obs. brățale (DEX16), *brăteele (DELR, s.v. braț), così come cordea, grădea, bretea, caramea, flanea, jartea rispetto a cordelă (< gr. kordélla), grădelă $(<\mathrm{sr}$. gradela), bretelă $\left(<\right.$ fr. bretelles) ${ }^{49}$, caramelă $(<$ fr. caramel/germ. Karamella/it. caramella), flanelă $(<\mathrm{fr}$. flanelle), jartelă $(<\mathrm{fr}$.jartelle). Per analogia in direzione

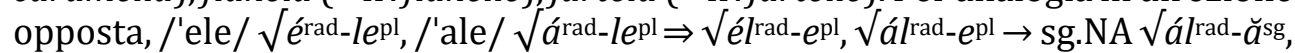

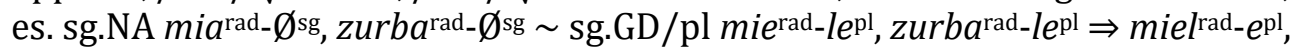
zurbalrad_epl, si spiegano le var. secondarie mială (con il senso specifico di 'ossicino d'agnello (usato in un gioco)'), zurbală, rispetto alle forme etimologiche mia ( $<$ lat. AGNĔLLA), zurba (< tc. zorba), così come le coppie năcăfală $\approx$ nacafa $(<\mathrm{tc}$. nafaka), sarmală $\approx$ sarma (< tc. sarma), zală $\approx$ za $(<\text { lat. < gr. zaba/sostr. })^{50}$.

Cimiliturile romanilor, 1898 (cf. MDA2, s.v. amețit ${ }^{1}$ ). L'assenza di forme di tipo *oprică per oprea (o in ogni caso la loro rarità, se ulteriori indagini dovessero confermarne l'esistenza) induce a ritenere che retrofomazioni da der. deverbali quali oprea tendano a essere assimilate più a s. primitivi quali acadea che a suffissati desostantivali e deaggettivali quali bucățea, albăstrea.

48 DEX16 e MDA2 sono concordi nel ritenere proptea e vopsea retroformazioni collegate ai der. F deverbali propteală, vopseală $\leftarrow$ v. propti (< sl.), vopsi (< gr.) + -eală, ma mentre MDA2 considera anche stricnea $\leftarrow$ pl stricnele $\sim$ sg stricneală, DEX16 lo dà $\leftarrow$ v. stricni $(<$ sl.) + suff. -ea. Lo statuto di -ea come suffisso deverbale non essendo chiaro (cf. n. precedente), seguo qui MDA2. Come retroformazioni di questo tipo si spiegano ottimamente anche oprea (che in quanto var. secondaria nei dizionari citati non ha etimologia propria) rispetto a opreală $\leftarrow$ v. opri $(<\mathrm{sl}$.) e podea (per cui sia DEX16 che MDA2 danno "etimologia sconosciuta") rispetto a podeală $\leftarrow$ v. podi $(\leftarrow$ s. N pod $<$ sl.).

49 In questo caso, in $\mathrm{r}$. standard le due varianti si sono specializzate semanticamente, bretea per la 'bretella' capo o parte di abbigliamento (DEX16) e raccordo stradale (D0OM2) e bretelă per la 'bretella' raccordo ferroviario (DEX16, DOOM2).

50 Questo tipo di retrofomazioni si è dimostrato particolarmente produttivo nel r. pop. e/o reg. (in particolare nelle parlate meridionali), tale che nel processo di normativizzazione alcune di esse (es. cordea, grădea, bretea) si sono imposte come var. primarie a scapito delle forme etimologiche. Verso la metà degli anni ' 40 del Novecento I. Iordan registrava la diffusione di forme quali bretea, caramea, momea (per momeală $\leftarrow$ v. momi < bg., sr.), pardosea (per pardoseală $\leftarrow$ v. pardosi $<$ ?) e santinea (per santinelă < fr. sentinelle), menzionando che alcune di esse, come bretea e sanda, fossero più utilizzate delle forme "regolari" (Iordan 1947, 103). Tale realtà appare debitamente 
È possibile che anche le var. in $\sqrt{a ́ i e ~(52 . c) ~ p o s s a n o ~ e s s e r e ~ r e t r o f o r m a z i o n i ~}$

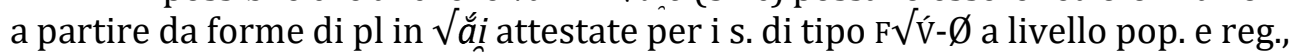
es. sg.NA manta 'cappa, mantella' pl lett. mantale, reg., pop. mantắi e mantắli $\left(G_{2} R_{2}, 80\right)^{51}$. Simili forme sono il prodotto dalla tendenza di lunga durata all'estensione del morfema $\mathrm{F}$ sg.GD/pl -i ai danni di sg.GD/pl -e, testimoniata da innumerevoli esempi nel passaggio dalla lingua antica a quella moderna e contemporanea (es. sg.NA limb-ă $\sim$ sg.GD/pl ant. limb-e $\rightarrow \bmod$. limb-i) e ancora attivo a livello pop. (es. sg.GD/pl pop. căș-i, plăss-i per lett. cas-e, plas-e $\sim$ sg.NA cas-ă, plas-ă; cf. anche i numerosi es. in Iordan 1947, 67-68), eventualmente accompagnata dall'estensione dell'alternanza fonetica sg.NA á $\sim \operatorname{sg} . \mathrm{GD} / \mathrm{pl} \stackrel{a}{a}$, specifica dei F con sg.GD/pl in -i (es. sg.NA banc-ă, cart-e $\sim$ sg.GD/pl bănc-i, cărtt-i; cf. anche sopra, sg.GD/pl pop. căș-i, plăș-i ${ }^{52}$, a sua volta accompagnata dall'eventuale alternanza secondaria sg.NA $a \sim \mathrm{sg}$.GD/pl $\breve{a}$ (meno frequente e a carattere prevalentemente pop.: es. sg talangă $\sim$ pl tălăngi, sg baracă, salată $\sim$ pl pop. bărăci, sălăți per lett. barăci, salate).

L'estensione parziale di F sg.GD/pl -i a F sg.GD/pl -le produce un nuovo morfema ibrido F sg.GD/pl -li /li / (cf. supra, §4.1.3, sg.GD mumbaieli): sg.NA sarmá- $\varnothing \sim \mathrm{sg} . \mathrm{GD} / \mathrm{pl}$ sarmá-le $\times-i \rightarrow \mathrm{sg} . \mathrm{GD} / \mathrm{pl}$ pop. sarmá-li53, sarmắ-li, laddove la sostituzione completa di F sg.GD/pl -le con F sg.GD/pl -i, regolarmente accompagnata dalla specifica alternanza innescata da questo morfema, genera forme quali sarmăi ${ }^{54}: \mathrm{sg} . \mathrm{GD} / \mathrm{pl}$ sarmá-le $+-i \rightarrow$ sg.GD/pl pop. sarmắ-i (cf. anche

registrata nei dizionari contemporanei, fino alle opere più recenti: nel caso della coppia di varianti sandală/sanda, ad es., in DOOM1 e fino a DEX98 la variante principale è stata l'etimologico sandală, mentre da DEX09 in poi è diventata sanda, questa essendo l'unica forma contemplata in DOOM2.

51 Pl quali măhălắli, mantắli (măn-), mantắi (măn-), sarmáli, sarmắli, sarmắi ecc. per lett. mahalále, mantále, sarmále ecc. (MDA2, s.v.) sono caratteristici delle parlate extracarpatice - già Tiktin $(1945 / 1883,52)$ menziona pl băsmăli, hărăbăli, bădănăli respingendoli come "provinciali" - e circolano ampiamente anche nella lingua scritta tra Ottocento e Novecento (cf. l'attestazione della frequenza di pl băsmăli, curăli ecc. in luogo dei "regolari" basmale, curele ecc. in Iordan $1947,69)$, con una certa presenza anche nella lingua letteraria di autori di origine in part. moldava, ad es. G. Bacovia (cf. il celebre verso "Prin măhălăli mai neagră noaptea pare...", Sonet), M. Sadoveanu, N. Iorga o Cezar Petrescu.

52 Tale alternanza compare anche nella formazione del $\mathrm{pl} \mathrm{F}$ in -uri, es. sg mâncar-e $\sim \mathrm{pl}$ mâncăr-uri, per via analogica ovvero "ereditata" da forme (cronologicamente anteriori) di sg.GD/pl in - $i$.

$53 \mathrm{Sg} . \mathrm{GD} / \mathrm{pl}$ di tipo sarmali /sar'mali/, risultanti da processi di natura morfologica, non vanno confusi con le forme prodotte dal fenomeno fonetico della chiusura di [e] atono a [i], che nella pratica corrente di letterarizzazione risultano omografe ma in cui la $i$ 〉 finale è vocalica $(e$ quindi sillabica): es. sg.GD/pl sarmale /sar'ma.le/ = [sar'ma.li], letterarizzato sarmali.

54 Questo tipo di livellamento analogico è particolarmente diffuso in ar., in cui il pl etimologico -le del tipo $\mathrm{F} \sqrt{\mathrm{V}}$-ŭă è stato sostituito nella maggior parte dei casi, compresi molti dei termini originari di eredità lat., da F pl -i: es. sg curá-ună, măseñ́-ună pl cură-i, măsé-i (vs sg steá-ună pl steńá-le). 
Guțu-Romalo 1968, 67). Dal confronto tra paradigmi di tipo sg.NA $\sqrt{a}$-ie sg.GD/pl $\sqrt{a}-i^{55}$, es. sg.NA cucuvá-ie $\sim$ sg.GD/pl cucuvă-i (cf. SDLR, IVOO 3 , DLRLC; forma etimologica < gr. koukouvágia) o sg.NA bá-ie, clá-ie $\sim$ sg.GD/pl bắ-i, clă-i, e sg.NA $\sqrt{a}-\varnothing \sim$ sg.GD/pl pop. $\sqrt{a}-i$, es. sg.NA trandâdá- $\varnothing \sim \operatorname{sg} . G D / p l$ pop. trandadă-i (lett. trandadá-le), si possono essere formati sg.NA analogici quali trandadái-e ${ }^{56}$, da un lato, e cucuveá-Ø (con il relativo sg.GD/pl cucuvé-le), dall'altro.

Un ulteriore tipo di retroformazione, le cui coppie non sono registrate nel corpus come var. morfologiche ma come lemmi indipendenti, è conseguente l'omonimia formale, simile a quelle sopra discusse, tra il sg.GD/pl di Fلécá- $\emptyset$ e il pl di s. $\mathrm{N}$ contenenti il suffisso o la sequenza sonora /'el/, es. N sg surcél- $\varnothing$ 'rametto' (< lat. *SURCĚLLU) pl surcél-e. Come nel caso precedente, la rianalisi di $\mathrm{N}$ pl surcélrad_epl $\Rightarrow \mathrm{F} \mathrm{pl}$ surcérad_lepl, resa possibile dal fatto che il "neutro" r. è di fatto un genus alternans, dal punto di vista dell'accordo $\mathrm{M}$ al sg e $\mathrm{F}$ al pl (cf. ad es. Maiden 2011 e 2015), ha portato alla formazione di un analogico F sg.NA surce árad_Øss57.

4.3.2. Formazioni diminutivali e variazione morfologica. Un caso particolare di variazione morfologica interclasse interessa i suffissati in -ea- $\varnothing$ (e composti, cf. supra, §4.2.2) e gli pseudoalterati in $\sqrt{e}$ ea- $\emptyset$ (qui di seguito indicati insieme come "(-)ea"), i quali conoscono nella maggior parte dei casi una forma sg.NA in -ică, che a partire dal XVIII sec. affianca e tende a soppiantare quella in (-)ea a causa della perdita di biunivocità tra forma e funzione suffissale di quest'ultimo, conseguente all'entrata in $r$. di numerosissimi turchismi adattati in $\sqrt{e a}$ (Florea 1984, 73-74; Maiden 1999).

Dei 71 formati a carattere diminutivale registrati nel corpus con forma anche o solo di sg poco più di un terzo, ovvero 27, sono lemmatizzati al sg.NA con duplice forma ea/ică o ică/ea (53.a), ai quali si aggiungono 11 pseudoalterati (53.b) ${ }^{58}$ :

55 Considero qui i $\mathrm{F}$ uscenti al sg.NA in /'aie/ come $\mathrm{F}$ in $-e$ e radice in á: sg.NA $* \sqrt{a}-e>\sqrt{a}-i e$ con inserzione di /i/ tra vocali al confine di morfema, e sg.GD/pl $* \sqrt{a}-i>\sqrt{a}-i$, con alternanza /'a/ $>$ /'ə/ e /i/ > /i/ dopo vocale.

56 Anche reg. sg dăndănaie pl dăndănăi "1. Tărăboi, tămbălău", che DEX16 e MDA2 cosiderano $\leftarrow$ fam. sg dandana pl dandanale "1. Întâmplare neplăcută; belea, bucluc, încurcătură" $(<$ tc. tantana) + -aie, potrebbe essere in realtà una retroformazione di questo tipo.

57 Molto prob. analogo il caso di F sg.NA căpețea 'cavezza' $\leftarrow$ N pl căpețele sg căpețel 'id.' (cf. supra, n. 29).

58 Do qui la var. in (-)ea sempre al primo posto, indicando in grassetto la var. considerata principale in DEX16 e D0OM2. In DEX16 tali coppie di var. sono lemmatizzate insieme con la var. in (-)ea al primo posto, secondo il modello "aluneá, -ícă"; eccezione fanno "pielicícă, -eá", "plămânărícă, -eá, mielușea e pătrățea, incluse entro i lemmi mielușél, -eá, -ícă e pătrățél, -eá, -ícă, e alcune coppie di var. date come lemmi separati con rivio: codăneá v. codănică, perceá v. percică, sălățeá v. sălățică, micșunícă v. micșunea, pătlăgícă v. pătlăgea, pingícă v. pingeá, ovvero come lemmi indipendenti, es. turtureá e turturícă, vițeá e vițícă. Rispetto a questo, DOOM2 promuove la var. 
(53) (a) alunea > alunică, bălticea > bălticică, brândușea < brândușică, cărticea > cărticică, codănea > codănică, floricea 〈floricică, găurea > găurică, jucărea > jucărică, lingurea ${ }^{1}$ < lingurică ${ }^{1}$, lingurea ${ }^{1}$ < lingurică ${ }^{2}$, măturea > măturică, mielușea < mielușică, minciunea < minciunică, miorea < miorică, părticea > părticică, păsărea > păsărică, pătrățea > pătrățică, picățea < picățică, pielicea > pielicică, plămânărea > plămânărică, pupezea 〈 pupezică, rămurea < rămurică, sălățea > sălătică, scândurea > scândurică, uscăturea < uscăturică, viorea < viorică, vișinea < vișinică;

(b) mărgea < mărgică, micșunea < micșunică, pătlăgea < pătlăgică, percea $>$ percică, pingea < pingică, purcea < purcică, surcea < surcică, turturea < turturică, ulcea < ulcică, vâlcea < vâlcică, vițea < vițică.

Di questi 38 s. con lemma plurivariante (che costituiscono quasi un decimo dell'intero corpus, per la precisione il 9,92\%), in DOOM2 23 s. (60,52\%) hanno come var. primaria la forma in (-)ea e $15 \mathrm{~s}$. quella in -ică, in quasi la metà dei casi $\left(7\right.$ s.) l'unica registrata ${ }^{59}$. Di fatto questi s. si inquadrano un ampio gruppo di suffissati diminutivali (o pseudo- tali) caratterizzati da forme di supplettivismo, per cui il suff. F -ică soppianta il suff. (o pseudo- tale) (-)ea60 creando forme di fatto sinonime ma con eventuali differenze d'uso e di registro (cf. Moroianu 2015). La sostituzione di (-)ea con -ică ha estensione diversa nel paradigma a seconda dei singoli lessemi (cf. già Carabulea 1977), e può avere luogo solo al sg.NA, in modo alternante (come nei casi sopra ricordati, per cui le fonti registrano una duplice forma di sg.NA: tipo A in 54) o esclusivo (tipo B), ovvero essere presente anche al sg.GD, in modo alternante (B1) o esclusivo (B2):

in -ică al primo posto per alunică, bălticică, cărticică, găurică, elimina la var. in (-)ea da 7 lemmi e promuove a lemma 1 var. in -ică (cf. n. successiva). Ho tenuto conto qui esclusivamente delle coppie $(-) e a \approx-i c a ̆$ presenti in DEX16 e/o DOOM2 in lemmi plurivariante o come lemmi separati ma collegati da rinvio lessicografico, mentre non ho incluso - ma rientrano a tutti gli effetti nello stesso discorso - quelle lemmatizzate sia in DOOM2 sia in DEX16 in modo indipendente (lemmi separati e assenza di rinvio), es. rândunică e rândunea, bucățică e bucățea ecc.

59 Un terzo delle var. in (-)ea (7 s.: jucărea, măturea, părticea, păsărea, pătrățea, percea, sălățea, scândurea) sono registrate solo in DEX16, DOOM2 contemplando come unica var. quella in -ică (jucărică ecc.); l'unico caso di var. in -ică di un s. in (-)ea presente in DEX16 e non presente in DOOM2 è pingică > pingea, così come vișinică è presente solo in D00M2 e non in DEX16.

$60 \mathrm{Da}$ un punto di vista diacronico che le cose siano andate in questo modo lo dimostrano il fatto che incontriamo varianti in -ică anche per s. in cui /'êna/ non è suffisso ma parte della radice, come in vari s. ereditati dal lat. o prestiti adattati (cf. supra), così come il fatto che in r. ant. le forme in (-)ea non presentano forme alternative in -ică (es. bucătea, fântânea), queste affermandosi successivamente al XVII sec. e in part. nelle parlate extracarpatiche (Florea 1984, 66, 76). Tuttavia, un gran numero di alterati di questo tipo possono non aver mai conosciuto il solo sg.NA in -ea, essendosi formati direttamente con il paradigma supplettivo una volta che questo si è consolidato. 
(54)

\begin{tabular}{|c|c|c|c|c|}
\hline & sg & pl & sg & pl \\
\hline & \multicolumn{2}{|c|}{ (A) } & \multicolumn{2}{|c|}{ (B) } \\
\hline NA & -ică / -ea & \multirow{2}{*}{-ele } & -ică & \multirow{2}{*}{-ele } \\
\hline GD & -ele & & -ele & \\
\hline & \multicolumn{2}{|c|}{ (B1) } & \multicolumn{2}{|c|}{ (B2) } \\
\hline NA & -ică & \multirow{2}{*}{$-e l e,-\boldsymbol{i c} \mathbf{i}$} & $-i c a ̆$ & \multirow{2}{*}{$\begin{array}{c}\text {-ele } \\
/ \text {-ici, -ele }\end{array}$} \\
\hline GD & -ele, -ici & & $-i c i$ & \\
\hline
\end{tabular}

Dei ca. 121 s. F suffissati a carattere dim. lemmatizzati in DOOM2 con la sola forma in -ică e che registrano almeno il sg.GD, 51 non presentano supplettivismo (55.a), mentre tra i restanti 70 - a fronte dei 27 dim. di tipo A sopra ricordati - si contano 57 lemmi di tipo B (55.b), 8 di tipo B1 (55.c) e 4 di tipo B2 (55.d):

(55) (a) non supplettivismo - antonică, ațică, băndurică, bibică, burtică, căldurică (fam.), fasolică (fam.), ferestrică (înv.), fetică (pop., fam.), fiicăa, frăsinică, fripturică, frunzică, frunzulică (pop.), gustărică, lânărică, lânică (pop.), lelică (pop.), levănțică, mămică (fam.), mămițică (fam.), mămulică (reg.), mătăsică, mândrulică (pop.), mierlică (rar), moșică (reg.), năfurică, nășică (pop.), negrilică, nevestică (fam.), nuvelică (rar), oițică (pop.), păpică (fam.), păpurică (reg.), pâinică, pânzică, plutică, poiețică (reg.), rândunică, roticică (rar), secărică (băutură), smântânică, sulițică (rar), timoftică, tușică (fam.), țăpossică (rar), țățică (reg.), verișorică (fam., rar), vulturică (rar), zambilică, zecică (fam.).

(b) tipo B - argințică, bavețică, bonețică, borticică (reg.), bucățică, burticică, căpșunică, ciuboțică, covățică (reg.), cumnățică (fam.), curticică, fecioricăa, firicică, flămânzică, flocoșică, foicică, frigurică, horboțică (reg.), iubițică (fam.), închegățică, jachețică, jucărică, lopățică, lumânărică, luntricică, maimuțică, măgurică (reg.), măturică, nepoțică, nopticică, olicică (pop.), păpușică, părticică, păturică, percică (reg.), picăturică (fam.), pielușică (reg.), pietricică, plăcințică (fam.), ploicică (fam.), prăjiturică (fam.), punticică (rar), putinică, răchițică, rourică (rar), săgețică, sălățică, săricică, scăricică, scrisorică, sforicică, sulițică (rar.), surățică (pop.), țăndărică ${ }^{1}$, vălicică (pop.), voinicică, zărzărică (pop.), zgrăbunțică;

(c) tipo B1 - broșurică (sg.GD/pl -ele/-ici), domnișorică (fam.) (sg.GD/pl-ele/-ici), filimică (sg.GD/pl-ele/-ici), legăturică (sg.GD/pl-ici/-ele), păsărică (sg.GD/pl -ici/-ele), trăsurică (sg.GD/pl -ici/-ele), tufănică (sg.GD/pl-ele/-ici);

(d) tipo B2 - băuturică (pl -ici/-ele), însemnărică (fam.) (pl -ele), jupâneșică (sg.GD.art -icăi/ichii, pl -ele), mătuşică (sg.GD.art -icăii/ichii/icii, pl -ele/-ici), mâncărică (fam.) (pl -ele). 
4.4. Conclusioni. Da un punto di vita morfologico, $F \sqrt{ } V^{-} \varnothing$ si distingue soprattutto per due tratti. Da un lato, il bassissimo numero di composti formati in r. che hanno come testa un s. appartenente a questa classe: nel corpus analizzato le creazioni di questo tipo si riducono a 3 sole parole, automacara, protostea, radiostea, tutti tecnicismi relativamente recenti e derivati da due tra le parole $\mathrm{F} \sqrt{\mathrm{V}}-\varnothing$ in assoluto più comuni e usate, stea 'stella' (< lat.) e macara 'gru' (macchina) $(<\mathrm{tc}$.).

Dall'altro, l'altissimo tasso di variabilità morfologica, in particolare interclasse. Un gran numero di s. appartenenti a questa classe hanno o sono var. secondarie appartenenti ad altre classi flessive o - in misura nettamente minore - a un altro sottotipo morfologico della classe stessa, frutto di un complesso gioco di adattamenti fonetici, rianalisi segmentali e analogie, e un numero altrettanto grande ha una var. secondaria o primaria in -ică, nel contesto di un vasto e duraturo processo di sostituzione con quest'ultimo del suffisso diminutivale -ea (ovvero del segmento radicale /'ea/ percepito come suff.), indebolito nelle sue funzioni diminutivali proprio dall'esistenza di una mole notevole di s. in $\sqrt{e} e$ non alterati. La variabilità morfologica di $F \sqrt{ }$ V́- $\emptyset$ nel corpus qui analizzato è tale - e una ricognizione di ulteriori fonti porterebbe alla luce un numero ancora maggiore di casi analoghi, di tipo fam. reg., pop. o obs. - da far ritenere che in r. standard contemporaneo essa sia nel complesso la classe flessiva con il più alto tasso di varianti morfologiche appartenenti ad altre classi flessive.

Tanto il basso numero di composti nuovi quanto l'alto numero di var. si spiegano, in sostanza, su base storica. Come si vedrà più chiaramente nel prossimo studio di questa serie, dedicato al profilo etimologico di $F \sqrt{v}-\varnothing$, questa classe è costituita in massima parte da prestiti relativamente recenti e in molti casi "periferici" rispetto al lessico fondamentale del r. (vuoi in quanto di per sé rari, vuoi perché storicismi ormai usciti dall'uso vivo, vuoi perché caratteristici dell'oralità e della colloquialità), e quindi, da un lato, attestati in diverse var. di adattamento al sistema fonomorfologico della lingua r. e, dall'altro, più esposti a pressioni analogiche. A parte stea, $z i$, termini di certa eredità latina per i quali la var. standard è affiancata nel corpus dalla var. reg. "lunga" (ed etimologica, cf. supra, tabella 38) steauă, ziuă, la variazione morfologica di cui al §4.3.1 riguarda infatti prestiti sette-ottocenteschi- da turco (la maggior parte), greco e francese. Le formazioni interne sono costituite quasi solo da derivati con il suff. dim. -ea, anch'esso però "messo in crisi" dall'entrata massiccia di prestiti adattati in /'ea/ e fortemente concorso da forme parallele più chiaramente dim. in -ică (cf. già Iordan, Guțu-Romalo, Niculescu 1967, 103).

Nonostante ciò, $F \sqrt{ } \hat{V}-\varnothing$ ha dato però anche prova di una relativa vitalità a livello fam. e pop., in particolare nelle parlate meridionali, testimoniata dalla creazione di nuove forme di tipo $\mathrm{F} \sqrt{\mathrm{V}}-\varnothing$ tramite derivazione regressiva, alcune delle quali sono state accettate come var. secondarie e persino primarie nella lingua standard: esemplare è il caso della recente accettazione nella norma attuale di sanda al posto di sandală (cf. supra, n. 50). 


\title{
ABBREVIAZIONI
}

\begin{tabular}{|c|c|c|}
\hline $\begin{array}{l}\text { (d)r. (daco)romeno } \\
\sqrt{\text { radic(alle }}\end{array}$ & $\begin{array}{l}\text { ir. istroromeno } \\
\text { it italiano }\end{array}$ & $\begin{array}{l}\text { reg. regionale } \\
\text { rs. russo }\end{array}$ \\
\hline a. aggettivo & lat. (dan.) latino & s. sostantivo \\
\hline ant. antico & (danubiano) & sg singolare \\
\hline ar. aromeno & м maschile & sl. slavo \\
\hline bg. bulgaro & mod. moderno & sost. sostrato \\
\hline der. derivato/i & mr. meglenoromeno & suff suffisso \\
\hline dim. diminutivo/i, & $\mathrm{N}$ neutro & tc. turco \\
\hline $\begin{array}{l}\text { diminutivale } / i \\
\text { F femminile }\end{array}$ & NA nominativo-accusativo & td. tedesco \\
\hline $\begin{array}{l}\text { F femminile } \\
\text { fam. familiare }\end{array}$ & pl plurale & v. verbo \\
\hline fr. francese & pop. popolare & var. variante/i \\
\hline $\begin{array}{l}\text { GD genitivo-dativo } \\
\text { gr. greco }\end{array}$ & $\begin{array}{l}\text { r.c. romeno comune } \\
\mathrm{rad} \text { radic(al)e }\end{array}$ & \\
\hline
\end{tabular}

\section{BIBLIOGRAFIA}

\begin{abstract}
A) DIZIONARI
CDER = Alexandru Ciorănescu, Dicționarul etimologic al limbii române. Ediție îngrijită și traducere din limba spaniolă de Tudora Șandru Mehedinți și Magdalena Popescu Marin, București: Saeculum I.0, 2007.

DDA = Tache Papahagi, Dicționarul dialectului aromân - general și etimologic, ediția a 2a augmentată, București: Editura Academia Republicii Socialiste România, 1974.

DELR = Marius Sala, Andrei Avram (dir.), Dicționarul etimologic al limbii române, III.1/II.2, București: Editura Academiei Române, 2012-2018.

DEX09 = Dicționarul explicativ al limbii române, ediția a 2a, revăzută și adăugită, București: Univers Enciclopedic, 2009.

DEX16 = Dicționarul explicativ al limbii romane, ediție revăzută și adăugită, București, Univers Enciclopedic Gold, 2016.

DEX98 = Dicționarul explicativ al limbii române, ediția a 2a, București: Univers Enciclopedic, 1998.

DLRLC = Dimitrie Macrea, Emil Petrovici (coord.), Al. Rosetti et al. (colectiv). Dicționarul limbii romîne literare contemporane, București: Editura Academiei Republicii Populare Române, 1955-1957.

D00M1 = Dicționarul ortografic, ortoepic și morfologic al limbii române, București: Editura Academiei Republicii Socialiste România, 1982.

D00M2 = Dicționarul ortografic, ortoepic și morfologic al limbii române, ediția a 2a revăzută și adăugită, București, Univers Enciclopedic Gold, 2010.

$\hat{I V O}_{3}=$ Sextil Pușcariu, Teodor A. Naum, Îndreptar și vocabular ortografic, ediția a IIIa, revăzută și completată, București; Editura „Cartea Românească”, 1941.
\end{abstract}


MDA = Marius Sala, Ion Dănăilă (dir.), Micul dicționar academic, IIV, București: Editura Univers Enciclopedic, 2001-2003.

PEW = Sextil Pușcariu, Etymologisches Wörterbuch der rumänischen Sprache. I: Lateinisches Element mit Berücksichtigung aller romanischen Sprachen, Heidelberg: K. Winter, 1905.

REW = Wilhelm Meyer Lübke. Romanisches Etymologisches Wörterbuch. Heidelberg, Carl Winters, 1935.

SDLR = August Scriban, Dicționaru limbii românești, București: Institutu de Arte Grafice „Presa Bună”, 1939.

TETT = Andreas Tietze, Tarihî ve etimolojik Türkiye Türkçesi lugati, TÜBA yayını editörü Semih Tezcan, IX, Ankara: TÜBA Türkiye Bilimler Akademisi, 2016-2020.

\section{B) TRATTATI, OPERE DI RIFERIMENTO, STUDI}

Agard, Frederick B. 1953. “Noun Morphology in Romanian.” In Language, 29(2), 134-42.

Borangic, Cătălin, Sorin Paliga. 2013. "Note pe marginea originii și a rolului armurilor getodacilor în ritualurile funerare." Acta Centri Lucusiensis 1A: 523, http://www.laurlucus.ro/actacentrilucusiensis/aclnr1a2013, data di ultima consultazione: 10/06/2021.

Carabulea, Elena. 1977. "Flexiunea derivatelor în IC $\breve{A}$ ”, in Limba română, 26(5), 569-74.

Coteanu, Ion, Angela Bidu-Vrănceanu, Narcisa Forăscu. 1985. Limba română contemporană: vocabularul. București: Editura Didactică și Pedagogică.

Coteanu, Ion. 1969. Morfologia numelui în protoromână (româna comună). București: Editura Academiei Republicii Socialiste România.

Coteanu. Ion. 2007. Formarea cuvintelor în limba română: derivarea, compunerea, conversiunea. București: Editura Universității din București.

Diaconescu, Paula. 1970. Structură și evoluție în morfologia substantivului românesc. București: Editura Academiei Republicii Socialiste România.

FCLR = Formarea cuvintelor în limba română, I. Compunerea, București: Editura Academiei Republicii Socialiste România, 1970; II. Prefixele, București: Editura Academiei Republicii Socialiste România, 1978; III. Sufixele. Derivarea verbală, București: Editura Academiei Republicii Socialiste România, 1989; IV.1. Sufixele. Derivarea nominală și adverbială. Partea 1, București: Editura Academiei Române, 2015.

Florea, Ion A. 1984. "Considerații diacronice asupra sufixului -ea." Anuar de lingvistică și istorie literară, XXIX (1983-1984), A. Lingvistică, 63-77.

Valeria Guțu Romalo (coord.). 2008. Gramatica limbii române. București: Editura Academiei Române.

Graur, Alexandru. 1975. Alte etimologii românești. București: Editura Academiei Republicii Socialiste România.

Guțu-Romalo, Valeria. 1968. Morfologia structurală a limbii române (substantiv, adjectiv, verb). București: Editura Academiei Republicii Socialiste România.

Marius Sala, Liliana Ionescu-Ruxăndoiu (coord.), Istoria limbii române, I, București, Univers Enciclopedic Gold, 2018.

Iordan, Iorgu, Valeria Guțu-Romalo, Alexandru Niculescu. 1967. Structura morfologică a limbii române contemporane. București: Editura Științifică. 
Maiden, Martin. 1999. "Il ruolo dell'«idoneità» in morfologia diacronica: i suffissi romeni -ea, -ică ed -oi." In Revue de linguistique romane, 63 (251--252), 321-345.

Maiden, Martin. 2011. Morphological persistence. In The Cambridge History of the Romance Languages. edited by Martin Maiden, John Charles Smith, Adam Ledgeway. Cambridge: Cambridge University Press, 155-215.

Maiden, Martin. 2015. Morfologia flexionară a pluralului românesc și așa-zisul 'neutru' în limba română și în graiurile românești. In Lucrările celui de-al cincilea Simpozion Internațional de Lingvistică, București, 27--28 septembrie 2013. edited by Marius Sala, Maria Stanciu Istrate, Nicoleta Petuho., București: Univers Enciclopedic Gold.

Merlo, Roberto. 2020, “Stelele și lalelele”: saggio di micromonografia storicodescrittiva di una classe flessiva della lingua romena (I)." Studia UBB Philologia 65, no. 4: 261280. DOI:10.24193/subbphilo.2020.4.16.

Moroianu, Cristian. 2007. Structură, proces și substituție în mecanismul derivării. In Limba română - stadiul actual al cercetării, edited by Gabriela Pană Dindelegan București: Editura Universității din București, 685-94.

Moroianu, Cristian. 2015. Sinonime analizabile. Studiu de caz: sinonimia diminutivală. In Caietele Sextil Pușcariu, II, Actele Conferinței Internaționale „Zilele Sextil Pușcariu”. Ediția a II-a, Cluj-Napoca, 10-11 septembrie 2015. ClujNapoca-: Scriptor/Argonaut, 294-313.

Noul atlas lingvistic român pe regiuni - Banat, VI.1. Material lingvistic necartografiat, București, Editura Academiei Române, in corso di stampa.

Neamțu, G.G., Cristina Bocoș, DianaMaria- Roman. 2019. Limba română contemporană. Caiete de seminar. Teoria și practica analizei fonetico--fonologice. Cluj--Napoca: Casa Cărții de Știință.

Nestorescu, Virgil. 2006. Din viața cuvintelor românești. București: Editura Academiei Române.

Pascu, Giorge. 1916. Sufixele românești. București: Librăriile Socec \& Company, C. Sfetea, P. Suru.

Șerban, Gabriela. 2009. Valori semantice și stilistice ale sufixului diminutival -el / -ea. In Lucrările celui de--al doilea Simpozion Internațional de Lingvistică, București, 28-29 noiembrie, 2008, edited by Nicolae Saramandu, Manuela Nevaci, Carmen Ioana Radu, București: Editura Universității din București, 331--38.

Șerban, Gabriela. 2010. "Sufixul diminutival -el / -ea, în limba română." Limba română, 59(4), 499--507.

Suciu, Emil. 2013, 101 cuvinte de origine turcă. Ediție elctonică. București: Humanitas.

Tiktin, Heimann. 1945 [1883]. Gramatica română. Etimologia și sintaxa. București: Tempo.

Vasiliu, Laura. 2001. Sufixe diminutivale. In Enciclopedia limbii române, edited by Mariu Sala, București: Univers Enciclopedic, 180-81.

Zafiu, Rodica. 2016. "De la împrumutătoare la ghilimici." Dilema veche, 635, https://dilemaveche.ro/sectiune/tilcshow/articol/delaimprumutatoarelaghilimici, data di ultima consultazione: 10/06/2021. 